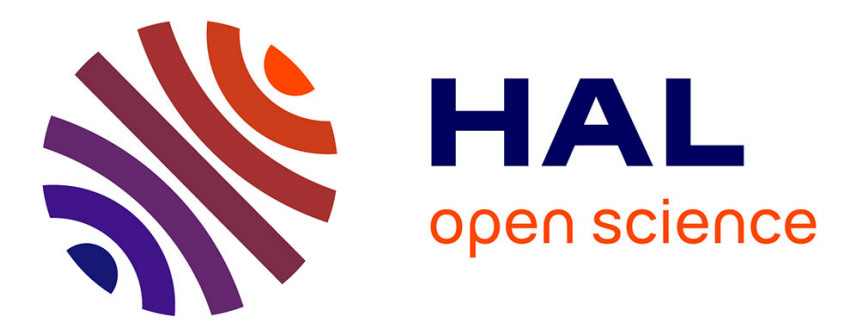

\title{
Global Lyapunov analysis of multi-stable nonlinear systems
}

Denis Efimov

\section{To cite this version:}

Denis Efimov. Global Lyapunov analysis of multi-stable nonlinear systems. SIAM Journal on Control and Optimization, 2012, 50 (5), pp.3132-3154. 10.1137/090767509 . hal-00745623

HAL Id: hal-00745623

https://hal.inria.fr/hal-00745623

Submitted on 26 Oct 2012

HAL is a multi-disciplinary open access archive for the deposit and dissemination of scientific research documents, whether they are published or not. The documents may come from teaching and research institutions in France or abroad, or from public or private research centers.
L'archive ouverte pluridisciplinaire HAL, est destinée au dépôt et à la diffusion de documents scientifiques de niveau recherche, publiés ou non, émanant des établissements d'enseignement et de recherche français ou étrangers, des laboratoires publics ou privés. 


\title{
GLOBAL LYAPUNOV ANALYSIS OF MULTI-STABLE NONLINEAR SYSTEMS
}

\author{
EFIMOV DENIS
}

\begin{abstract}
A new type of stability is introduced and its equivalent Lyapunov characterization is presented. The problem of global stability for the compact set composed of all invariant solutions of a nonlinear system (several equilibriums, for instance) is studied. Such problem statement allows us to analyze global stability properties for multi-stable systems. It is shown that several well-known multi-stable systems satisfy this new stability property.
\end{abstract}

\section{INTRODUCTION}

The problem of stability of nonlinear systems is one of the main issues in dynamical system theory. The main attention is paid to local or global stability of equilibriums or trajectories [11, 13, 14, 17, 24], set stability [16], stability with respect to part of variables [23,34], robust stability in presence of exogenous inputs $[27,30,29]$ and oscillation analysis $[4,8,31]$. An interest to multi-stability is also growing during the last decade $[1,2,3,7,22]$. Multi-stable systems include bistable ones (the class of systems with at least two stable equilibriums), almost globally stable systems (which have one attracting invariant set and the rest are repellers) and nonlinear systems with generic invariant sets. Each invariant solution or equilibrium can be analyzed locally applying a conventional stability paradigm. The multi-stability phenomenon arises when it is necessary to analyze behavior of a system globally, taking into account all its possible final states and motions. One of the main areas of application of the multi-stability framework is the theoretical biology (the models of cell differentiation, cell cycle and circadian oscillators, apoptosis and many others have several compact invariant sets or equilibriums [15]). Another traditional area is mechanics (Duffing equation, Van der Pol equation, Hamiltonian systems, under some conditions they all have several invariant solutions and different number of stable equilibriums [12]).

There are several approaches appealing to multi-stability problem. The first monograph devoted to this subject was published over 30 years ago [10]. The problem of Lyapunov function existence for multi-stable systems on a compact manifold has been studied in $[35,20,19]$. One popular modern approach is based on density functions $[1,33,18,22]$. This approach assumes the existence of a single asymptotically stable equilibrium or invariant set and the presence of several unstable ones. To establish stability of the attracting set, this approach substitutes the conventional condition on Lyapunov function existence with another one involving a density function. The obtained property is called almost global stability since it excludes from consideration the set of initial conditions with zero measure (attraction is guaranteed for all initial conditions except a set with zero Lebesgue measure). This approach cannot be applied to a system with several attracting subsets and it 
could be rather hard to find a density function for a particular example. Another line of research deals with analysis of monotone systems, which are widely used in biology. Multi-stability of this type of models is addressed in the papers [2, 3, 4, 9], where several constructive conditions for equilibrium stability have been developed. In the papers $[7,31]$ bistable systems are considered for a particular form of equations, which have two stable invariant sets with right hand sides described by a full state linear negative feedback plus bounded nonlinearities, each stable set is characterized by local input-to-state stability. In the paper [21] a Lyapunov density based notion of almost everywhere stability is introduced. This approach is based on solution of a partial differential equation.

The present paper aims to add another solution in the palette of approaches dealing with the multi-stability. Contrarily the previously mentioned works, which mainly deal with local stability of an invariant solution or almost global stability of the single stable set, in this work a global asymptotic stability notion for multi-stable systems is proposed. The set of all invariant solutions of the system (including locally stable or unstable ones) is chosen as the object of investigation. The obtained solution is inspired by biological and mechanical systems discussed in the next section. Section 3 contains definition of the new stability notion. Section 4 presents the necessary and sufficient Lyapunov characterization. Some examples are considered in section 5 .

\section{Motivating EXAMPles}

Consider Lotka-Volterra equations, also known as the predator-prey equations:

$$
\begin{aligned}
& \dot{x}=\alpha x-\beta x y ; \\
& \dot{y}=-\alpha y+(\delta+u) x y,
\end{aligned}
$$

where $x>0$ is the number of preys, $y>0$ is the number of predators, $\alpha, \beta, \delta>0$ are parameters representing interactions between these two species, $u \in \mathbb{R}$ is the control input ensuring the desired population dynamics. For the case $u=0$ the system (1) has Hamiltonian function

$$
H(x, y)=\delta x+\beta y-\alpha \ln (x y)
$$

with the minimum $H_{0}=\alpha\left[2-\ln \left(\alpha^{2} /\{\beta \delta\}\right)\right]$ at the unique equilibrium of the system $(\alpha / \delta, \alpha / \beta)$, the rest trajectories of the unforced system (1) are closed orbits. Tacking the control input

$$
u=k(\alpha-\beta y) x\left[H(x, y)-H_{d}\right],
$$

where $H_{0}<H_{d}<+\infty$ is the desired level of Hamiltonian function and $k>0$ is a parameter, it is possible to asymptotically stabilize the particular system orbit corresponding to $H_{d}$. Therefore, the set of all invariant solutions of the system is given by $\mathcal{W}=\{(\alpha / \delta, \alpha / \beta) \cup \Gamma\}$, where $\Gamma=\left\{(x, y): H(x, y)=H_{d}\right\}$. The system (1) has Lyapunov function $W(x, y)=0.5\left[H(x, y)-H_{d}\right]^{2}$, whose time derivative can be presented as follows:

$$
\dot{W}=-k y^{2}, y=(\alpha-\beta y) x\left[H(x, y)-H_{d}\right] .
$$

Boundedness of $W$ implies boundedness of the state $(x, y)$. We know from the Krasovskii-LaSalle invariance principle that all trajectories converge to the set $\mathcal{Z}=\{(x, y): y=0\}$. Since $\mathcal{W} \subset \mathcal{Z}$ and the set $\mathcal{W}$ contains all invariant solutions of the system, the global attractiveness of $\mathcal{W}$ is ensured. Trajectories of the system and contours of the Lyapunov function are presented in Fig. 1. Since the 

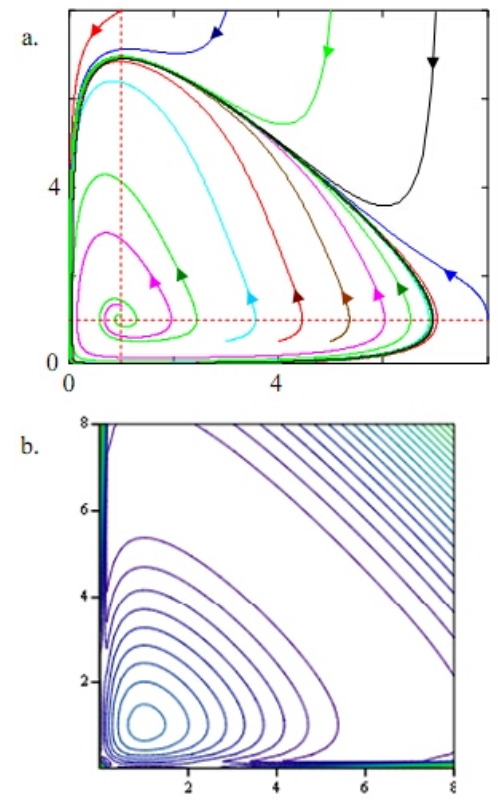

FiguRE 1. Trajectories and Lyapunov function contours for the system (1)

equilibrium is unstable (for $\alpha=\beta=\delta=1, k=0.1$ the system linearization has two complex conjugate eigenvalues with positive real parts in the equilibrium) almost all trajectories converge to the limit cycle $\Gamma$. A peculiarity of this example is that many other controlled Hamiltonian systems have a similar function $W$.

The second example is the Duffing system:

$$
\begin{gathered}
\dot{x}_{1}=x_{2} ; \\
\dot{x}_{2}=-x_{2}+x_{1}-x_{1}^{3}, x_{1} \in R, x \in R .
\end{gathered}
$$

This system has three equilibriums with coordinates $(0,0),(-1,0),(0,1)$. Linearization of the system in these points shows that the first equilibrium at the origin is a saddle point (one positive and one negative real eigenvalues) and the last two are locally asymptotically stable (complex conjugated eigenvalues with negative real parts). It is a good example of bistable systems, all trajectories converge to one of the stable equilibriums depending on initial conditions. The set of all invariant solutions of this systems $\mathcal{W}$ is composed by these equilibriums, i.e., $\mathcal{W}=\{(0,0),(-1,0),(0,1)\}$. This set is globally attractive (see Fig. 2,a). The system (2) has Lyapunov function

$$
W\left(x_{1}, x_{2}\right)=0.25\left(x_{1}^{4}-2 x_{1}^{2}+2 x_{2}^{2}\right)+0.25,
$$

which equals zero in the stable equilibriums only and it is positive otherwise (the function $W$ contour plot is presented in Fig. 2,b). Its time derivative for the system (2) is not positive:

$$
\dot{W}=-y^{2}, y=x_{2} .
$$

By the same Krasovskii-LaSalle arguments all trajectories are bounded and converge to the set $\mathcal{W}$. The stable equilibriums attract trajectories for almost all 

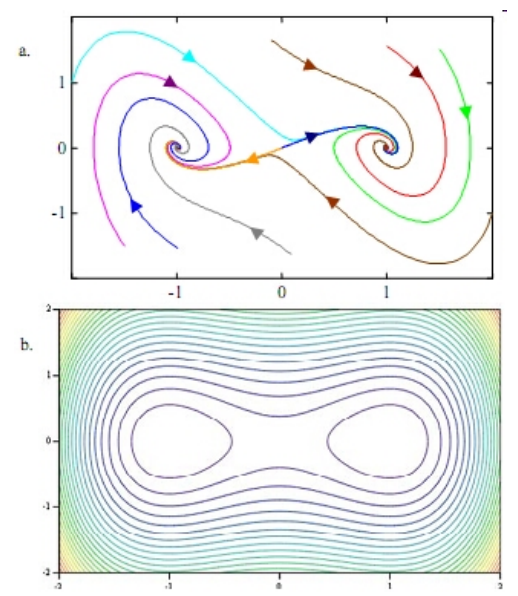

Figure 2. Trajectories and Lyapunov function contours for the system (2)

initial conditions, the exceptions are the equilibrium at the origin and the stable separatrices of this equilibrium.

The presented systems are multi-stable and possess some special non strict Lyapunov functions (the functions equal zero on the attracting limit set and are positive otherwise, their time derivatives are negative semidefinite). Let us generalize these examples and propose a stability property with respect to such a set $\mathcal{W}$ characterized by the observed Lyapunov functions.

\section{Stability Definition}

The section starts with preliminaries. In the second subsection the definition of stability is presented and discussed. In the third subsection it is shown that under some mild conditions, nonlinear systems satisfy the proposed stability property with respect to the set of all invariant solutions. In the last subsection, some useful upper estimates on such a system solutions are established.

3.1. Preliminaries. To deal with the systems presented in the motivating examples, it is necessary to consider nonlinear systems evolving on a manifold:

$$
\dot{\mathbf{x}}=\mathbf{f}(\mathbf{x})
$$

where $\mathbf{x} \in M, \operatorname{dim}(M)=n$ is the state and $M$ is a smooth manifold with a metric $d: M \times M \rightarrow \mathbb{R}_{+}, \mathbb{R}_{+}=\{s \in \mathbb{R}: s \geqslant 0\} ; \mathbf{f}: M \rightarrow T M$ is a locally Lipschitz manifold map. For all initial conditions $\mathbf{x}_{0} \in M$, the corresponding solution is denoted $\mathbf{x}\left(t, \mathbf{x}_{0}\right)$ (the short notation $\mathbf{x}(t)$ is used if origin of initial conditions is apparent). The solutions are unique, continuous and defined at least locally in time. If the trajectories $\mathbf{x}\left(t, \mathbf{x}_{0}\right)$ are defined for all $\mathbf{x}_{0} \in M$ and $t \geq 0$, then the system is called forward complete (further we will always assume that (3) is forward complete). Denote for any point $\mathbf{x} \in M$ the norm $|\mathbf{x}|=d\left(\mathbf{x}, 0_{M}\right)$, where $0_{M}$ is "the origin" on the manifold $M$, then for any set $\mathcal{A} \subset M$ the distance to the set from a point $\mathbf{x} \in M$ is defined as $|\mathbf{x}|_{\mathcal{A}}=\inf _{\xi \in \mathcal{A}}|\mathbf{x}-\xi|$.

A set $\mathcal{A} \subset M$ is called forward invariant for the trajectories of the system (3) if $\mathbf{x}\left(t, \mathbf{x}_{0}\right) \in \mathcal{A}$ for all $\mathbf{x}_{0} \in \mathcal{A}$ and $t \geq 0$; a set $\mathcal{A} \subset M$ is called backward invariant for 
the trajectories of the system (3) if $\mathbf{x}\left(t, \mathbf{x}_{0}\right) \in \mathcal{A}$ for all $\mathbf{x}_{0} \in \mathcal{A}$ and $t \leq 0$; and a set $\mathcal{A} \subset M$ is invariant if it is forward and backward invariant simultaneously for (3). For the system (3) an invariant set $\mathcal{A} \subset M$ is locally attracting if there exists an open neighborhood $\mathcal{X} \subset M, \mathcal{A} \subset \mathcal{X}$ such that $\varlimsup_{t \rightarrow+\infty}\left|\mathbf{x}\left(t, \mathbf{x}_{0}\right)\right|_{\mathcal{A}}=0$ for all $\mathbf{x}_{0} \in \mathcal{X}$; an invariant set $\mathcal{R} \subset M$ is locally repelling if there exists an open neighborhood $\mathcal{X} \subset M, \mathcal{R} \subset \mathcal{X}$ such that $\varlimsup_{t \rightarrow-\infty}\left|\mathbf{x}\left(t, \mathbf{x}_{0}\right)\right|_{\mathcal{R}}=0$ for all $\mathbf{x}_{0} \in \mathcal{X}$.

An equilibrium of (3) $\mathbf{x}_{0} \in M$ is called hyperbolic if the matrix $\mathbf{A}_{\mathbf{x}_{0}}=\partial \mathbf{f}(\mathbf{x}) /\left.\partial \mathbf{x}\right|_{\mathbf{x}=\mathbf{x}_{0}}$ has $k$ eigenvalues with positive real part and $n-k$ eigenvalues with negative real part, $0<k<n$ ( $n$ is the manifold dimension). In neighborhood of a hyperbolic equilibrium there exist $n-k$-dimensional invariant submanifold $S_{\mathbf{x}_{0}}^{+}$and $k$ dimensional invariant submanifold $S_{\mathbf{x}_{0}}^{-}$such that

$$
\varlimsup_{t \rightarrow+\infty}\left|\mathbf{x}(t, \xi)-\mathbf{x}_{0}\right|=0, \varlimsup_{t \rightarrow-\infty}\left|\mathbf{x}(t, \zeta)-\mathbf{x}_{0}\right|=0
$$

for all $\xi \in S_{\mathbf{x}_{0}}^{+}$and $\zeta \in S_{\mathbf{x}_{0}}^{-}$. An invariant set $\mathcal{H} \subset M$ is called locally hyperbolic if for any $\mathbf{x}_{0} \in \mathcal{H}$ the point $\mathbf{x}_{0}$ is a hyperbolic equilibrium (then $\mathbf{x}_{0}$ is an isolated equilibrium) or the tangent space $T_{\mathbf{x}_{0}} M$ can be decomposed into a direct (Whitney) sum $T_{\mathbf{x}_{0}} M=E_{\mathbf{x}_{0}}^{+} \oplus E_{\mathbf{x}_{0}}^{-} \oplus E_{\mathbf{x}_{0}}^{0}\left(E_{\mathbf{x}_{0}}^{0}\right.$ is spanned by phase velocity vector and defines movement of the trajectory $\mathbf{x}\left(t, \mathbf{x}_{0}\right)$ in the direction of the set $\left.\mathcal{H}\right)$. The following inequalities have to be satisfied for all $\xi \in E_{\mathbf{x}_{0}}^{+}$and $\zeta \in E_{\mathbf{x}_{0}}^{-}$:

$$
\begin{gathered}
\underline{C} \lambda^{t}|\zeta| \leqslant\left|D \mathbf{x}\left(t, \mathbf{x}_{0}\right) \zeta\right| \leqslant \bar{C} \lambda^{t}|\zeta|, \\
\underline{C} \lambda^{-t}|\xi| \leqslant\left|D \mathbf{x}\left(t, \mathbf{x}_{0}\right) \xi\right| \leqslant \bar{C} \lambda^{-t}|\xi|, t \geq 0
\end{gathered}
$$

for some $0<\underline{C}<\bar{C}, 0<\lambda<1$, where $D \mathbf{x}\left(t, \mathbf{x}_{0}\right)$ denotes the trajectory $\mathbf{x}\left(t, \mathbf{x}_{0}\right)$ differential $[5,6,26]$. For any $\mathbf{x}_{0} \in \mathcal{H}$ the stable and unstable invariant submanifolds $S_{\mathbf{x}_{0}}^{+}$and $S_{\mathbf{x}_{0}}^{-}$can be defined in a neighborhood of the point $\mathbf{x}_{0}$ in a similar way. Let

$$
\mathcal{H}^{+}=\bigcup_{\mathbf{x}_{0} \in \mathcal{H}} S_{\mathbf{x}_{0}}^{+}, \mathcal{H}^{-}=\bigcup_{\mathbf{x}_{0} \in \mathcal{H}} S_{\mathbf{x}_{0}}^{-},
$$

then $\mathcal{H}=\mathcal{H}^{+} \cap \mathcal{H}^{-}$.

A closed invariant set $\mathcal{A} \subset M$ is called minimal if it does not contain other smaller invariant sets. Further talking about invariants of the system (3) we always assume that the invariant sets are closed and minimal.

\subsection{Stability property.}

Definition 1. For a set $\mathcal{W} \subset M$ :

- the system (3) is $\mathcal{W}$-stable if for some given $R \geqslant 0$ and for any $R \leqslant \varepsilon<+\infty$ there exists $0 \leqslant \delta<+\infty$ such that $\left|\mathbf{x}\left(t, \mathbf{x}_{0}\right)\right|_{\mathcal{W}} \leqslant \varepsilon$ for all $t \geqslant 0$ whenever $\left|\mathbf{x}_{0}\right|_{\mathcal{W}} \leqslant \delta$;

- the system (3) is $\mathcal{W}$-attracting if for any $0<\varepsilon<+\infty$ and $\mathbf{x}_{0} \in M$ there exists $0 \leqslant T_{\mathbf{x}_{0}, \varepsilon}<+\infty$ such that $\left|\mathbf{x}\left(t, \mathbf{x}_{0}\right)\right| \mathcal{W} \leqslant \varepsilon$ for all $t \geqslant T_{\mathbf{x}_{0}, \varepsilon}$.

- the system (3) is $\mathcal{W}$-asymptotically stable if it is $\mathcal{W}$-stable and $\mathcal{W}$-attracting.

The properties introduced in definition 1 differ from conventional Lyapunov stability properties $[17,16]$ in the following aspects. First, $\mathcal{W}$-stability is introduced for $\varepsilon \geqslant R$ for some $R \geqslant 0$ (for $R=0$ the $\mathcal{W}$-stability property is reduced to the conventional Lyapunov stability), the presence of $R$ implies that setting initial point of a trajectory in some infinitesimal vicinity of the set $\mathcal{W}$ with $\delta \rightarrow 0$ does not ensure location of the trajectory in this vicinity. The case $R>0$ corresponds to possible "unstable" equilibriums inclusion into the set $\mathcal{W}$, when the trajectories 
a.

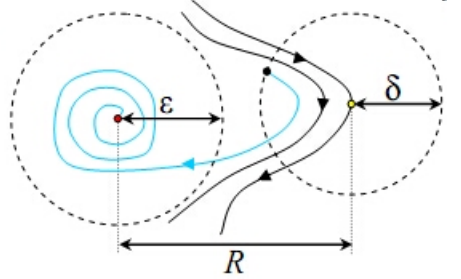

b.

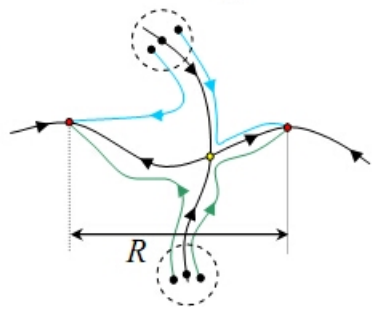

FiguRE 3. Illustration on the stability property definition

initiated close to these equilibriums have to exit neighborhoods of unstable equilibriums converging to stable ones. As an illustration of such behavior see Fig. 3,a, where the colored trajectory starting in $\delta$-vicinity of a hyperbolic equilibrium goes to $\varepsilon$-vicinity of the attracting fixed point. Following the illustration, the constant $R$ can be related to the radius of the set $\mathcal{W}$, i.e., with $\sup _{\mathbf{x}_{1}, \mathbf{x}_{2} \in \mathcal{W}}\left|\mathbf{x}_{1}-\mathbf{x}_{2}\right|$. In Fig. $3, \mathrm{~b}$ another situation is presented, when the colored trajectories closely initiated to separatrices converge to different attracting states. The left and right distances from the separatrices to the attracting states can differ significantly, which again indicates the necessity of the constant $R$ introduction, giving flexibility for the trajectories local behavior near the equilibriums. Second, according to definition 1 , for any $r>0$ it may be

$$
T_{r, \varepsilon}=+\infty, T_{r, \varepsilon}=\sup _{\left|\mathbf{x}_{0}\right| \mathcal{W}<r} T_{\mathbf{x}_{0}, \varepsilon}
$$

In other words, there is no uniform time of convergence to the set $\mathcal{W}$ for compact sets of initial conditions. This property is the result of "unstable" equilibriums/sets presence also.

Recall that a continuous function $\mu: \mathbb{R}_{+} \rightarrow \mathbb{R}_{+}$belongs to class $\mathcal{K}$ if $\mu(0)=0$ and it is strictly increasing; additionally it is of class $\mathcal{K}_{\infty}$ if it is also radially unbounded; a continuous function $\beta: \mathbb{R}_{+} \times \mathbb{R}_{+} \rightarrow \mathbb{R}_{+}$is of class $\mathcal{K} \mathcal{L}$, if $\beta(\cdot, t) \in \mathcal{K}$ for any $t \in \mathbb{R}_{+}$, and $\beta(s, \cdot)$ is strictly decreasing to zero for any fixed $s \in \mathbb{R}_{+}$. Applying standard arguments the following more useful equivalent characterization of $\mathcal{W}$-stability for the system (3) can be obtained.

Proposition 1. The system (3) is $\mathcal{W}$-stable for the given $R \geqslant 0$ if and only if there exists a function $\phi \in \mathcal{K}$ such that $\left|\mathbf{x}\left(t, \mathbf{x}_{0}\right)\right|_{\mathcal{W}} \leqslant \phi\left(\left|\mathbf{x}_{0}\right|_{\mathcal{W}}+R^{\prime}\right), R^{\prime}=\phi^{-1}(R), t \geqslant 0$ for all $\mathbf{x}_{0} \in M$.

Proof. To prove sufficiency, denote $\varepsilon=\phi\left(\delta+R^{\prime}\right)$ for any $\delta \geqslant 0\left(\varepsilon \geqslant \phi\left(R^{\prime}\right)=R\right)$, then the $\mathcal{W}$-stability holds.

Necessity. From definition 1 , for a given $R \geqslant 0$ and for any $\varepsilon \geqslant 0$ there exists $\delta \geqslant 0$ such that $\left|\mathbf{x}\left(t, \mathbf{x}_{0}\right)\right|_{\mathcal{W}} \leqslant R+\varepsilon$ for all $t \geqslant 0$ whenever $\left|\mathbf{x}_{0}\right|_{\mathcal{W}} \leqslant \delta$. For all $s>0$ define a function $g(s)=\sup _{\left|\mathbf{x}_{0}\right|_{\mathcal{W}} \leqslant s, t \geqslant 0}\left|\mathbf{x}\left(t, \mathbf{x}_{0}\right)\right|_{\mathcal{W}}$ and let $g(0)=R$, then 
$g(s)<+\infty$ for any $s \in \mathbb{R}_{+}$(by definition of $\mathcal{W}$-stability) and $g\left(s_{1}\right) \leqslant g\left(s_{2}\right)$ for $s_{1} \leqslant s_{2}$. From the definition, for all $\varepsilon \geqslant 0$ there exists $\delta \geqslant 0$ such that $g(\delta) \leqslant R+\varepsilon$, then for any $\varepsilon^{\prime}>0$ there exists $\delta^{\prime}>0$ such that $\left|g\left(s^{\prime}\right)-g(s)\right| \leqslant \varepsilon^{\prime}$ whenever $\left|s^{\prime}-s\right| \leqslant \delta^{\prime}$ for all $s^{\prime} \in \mathbb{R}_{+}$. Therefore, the function $g$ is not decreasing and continuous. Then there exists a function $\phi$ from class $\mathcal{K}$ yielding $g(s) \leqslant \phi\left(s+R^{\prime}\right)$, $R^{\prime}=\phi^{-1}(R)$, which is required.

The conventional Lyapunov stability with respect to an invariant set $\mathcal{W}$ can be introduced as follows [16]: for any initial conditions $\mathbf{x}_{0} \in M$, there exists a function $\delta \in \mathcal{K}$ such that $\left|\mathbf{x}\left(t, \mathbf{x}_{0}\right)\right|_{\mathcal{W}} \leqslant \delta\left(\left|\mathbf{x}_{0}\right| \mathcal{W}\right)$ for all $t \geqslant 0$. Thus the deviations of trajectories from the set $\mathcal{W}$ are proportional to the initial divergence from the set. According to proposition 1, in the $\mathcal{W}$-stability case, the deviations are proportional to the initial ones subject to a shift $R^{\prime}$ (similarly the practical stability with respect to the set $\mathcal{W}$ can be formulated in the conventional framework [16]). If the set $\mathcal{W}$ is not connected and contains stable and unstable subsets, then the constant $R$ characterizes the distance between the subsets, i.e. locally trajectory can exit any vicinity of an unstable subset and enters the stable ones. However, globally (when $\left|\mathbf{x}_{0}\right|_{\mathcal{W}} \gg R^{\prime}$ ) the trajectories deviations are governed by the initial divergence, similarly to the conventional Lyapunov stability.

3.3. Stability with respect to set of all invariant solutions. Let $\mathcal{W} \subset M$ be a compact non empty set containing all invariant sets of the system (3) (the set $\mathcal{W}$ is not necessarily connected). Let $\varlimsup_{t \rightarrow+\infty}\left|\mathbf{x}\left(t, \mathbf{x}_{0}\right)\right|_{\mathcal{W}}=0$ for all $\mathbf{x}_{0} \in M$.

The global attractiveness of the set $\mathcal{W}$ excludes from consideration the systems with unbounded solutions, additionally this assumption prevents some configurations of invariant sets obeying separatrices with solutions approaching infinity. Due to compactness of the set $\mathcal{W}$ there is a constant $D \geqslant 0$ (related with $\left|0_{M}\right| \mathcal{W}$ ) such that

$$
|\mathbf{x}|_{\mathcal{W}} \leqslant \mu_{1}(|\mathbf{x}|+D),|\mathbf{x}| \leqslant \mu_{2}\left(|\mathbf{x}|_{\mathcal{W}}+D\right), \mu_{1}, \mu_{2} \in \mathcal{K} .
$$

Further we assume that the manifold $M$ has the property $\sup _{\mathbf{x} \in M}|\mathbf{x}|_{\mathcal{W}}=+\infty$.

For the set $\mathcal{W}$ formed by all invariants of the system (3), the property of $\mathcal{W}$ asymptotic stability is naturally satisfied.

Lemma 1. Let $\mathcal{W} \subset M$ be a compact set containing all invariant sets of the system (3) and

$$
\overline{\lim }_{t \rightarrow+\infty}\left|\mathbf{x}\left(t, \mathbf{x}_{0}\right)\right|_{\mathcal{W}}=0
$$

for all $\mathbf{x}_{0} \in M$. Then there exists $R \geqslant 0$ such that the system (3) is $\mathcal{W}$-asymptotically stable.

Proof. The set $\mathcal{W}$ contains all invariant solutions and $\varlimsup_{t \rightarrow+\infty}\left|\mathbf{x}\left(t, \mathbf{x}_{0}\right)\right|_{\mathcal{W}}=0$ for all $\mathbf{x}_{0} \in M$, then for each such $\mathbf{x}_{0}$ for all $\varepsilon>0$ there exists a finite time $T_{\mathbf{x}_{0}, \varepsilon} \geqslant 0$ such that $\left|\mathbf{x}\left(t, \mathbf{x}_{0}\right)\right|_{\mathcal{W}}<\varepsilon$ for all $t \geqslant T_{\mathbf{x}_{0}, \varepsilon}$ (if a point $\mathbf{x}_{0} \in M \backslash \mathcal{W}$ satisfies the property $T_{\mathbf{x}_{0}, \varepsilon}=+\infty$ for some $\varepsilon>0$, then the corresponding trajectory $\mathbf{x}\left(t, \mathbf{x}_{0}\right)$ does not approach the set $\mathcal{W}$ with $t \rightarrow+\infty$, therefore $\mathbf{x}\left(t, \mathbf{x}_{0}\right)$ has its own invariant set, that is a contradiction), thus the system is $\mathcal{W}$-attracting.

Next, consider the set of initial conditions $\mathbf{x}_{0} \in D_{\delta}$ for some $\delta \in \mathbb{R}_{+}, D_{\delta}=$ $\left\{\mathbf{x} \in M:|\mathbf{x}|_{\mathcal{W}}<\delta\right\}$. Define $n_{\mathbf{x}_{0}}=\sup _{t \geqslant 0}\left|\mathbf{x}\left(t, \mathbf{x}_{0}\right)\right|_{\mathcal{W}}, n_{\mathbf{x}_{0}} \geqslant\left|\mathbf{x}_{0}\right|_{\mathcal{W}}$ and $N_{\delta}=$ $\sup _{\mathbf{x}_{0} \in D_{\delta}} n_{\mathbf{x}_{0}}$. Assume that $N_{\delta^{\prime}}=+\infty$ for some finite $\delta^{\prime} \in \mathbb{R}_{+}$, it means existence of a sequence $\mathbf{x}_{i} \in D_{\delta^{\prime}}, i \geqslant 0$ with trajectories $\mathbf{x}\left(t, \mathbf{x}_{i}\right)$ such that $\sup _{i \geqslant 0} \sup _{t \geqslant 0}\left|\mathbf{x}\left(t, \mathbf{x}_{i}\right)\right|_{\mathcal{W}}=$ 
a.

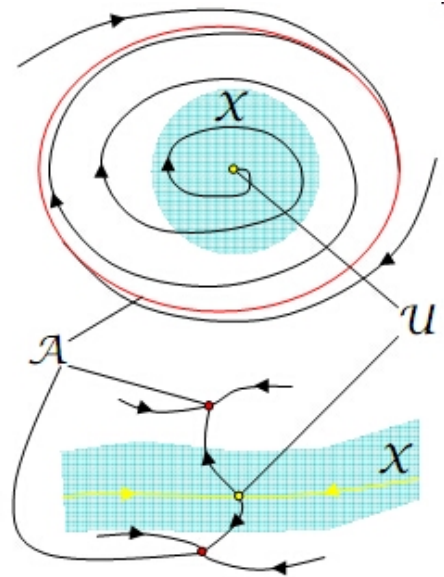

FIGURE 4. Illustration to facts 1 and 2

$+\infty$. Take $0<\varepsilon \leqslant \delta$, then there exists $0 \leqslant T_{\mathbf{x}_{i}, \varepsilon}<+\infty, i \geqslant 0$ such that $\left|\mathbf{x}\left(t, \mathbf{x}_{i}\right)\right|_{\mathcal{W}}<\varepsilon$ for all $t \geqslant T_{\mathbf{x}_{i}, \varepsilon}$ and all $\mathbf{x}_{i} \in D_{\delta^{\prime}}$. Therefore, the trajectories $\mathbf{x}\left(t, \mathbf{x}_{i}\right)$ approach infinity and return back in a finite time ( $T_{\mathbf{x}_{i}, \varepsilon}$ is an upper bound). However due to compactness of $\mathcal{W}$ and continuity of the solutions, the norms $\left|\mathbf{x}\left(t, \mathbf{x}_{i}\right)\right|$ stay bounded and each particular trajectory has finite norm (the system is forward complete). For the sequence of trajectories this implies the existence of a limit point $\mathbf{x}_{\infty}$ on the boundary of the set $D_{\delta^{\prime}}$ with a trajectory $\mathbf{x}\left(t, \mathbf{x}_{\infty}\right)$ approaching infinity and returning back in a finite time $T_{\mathbf{x}_{\infty}, \varepsilon}$, which is a contradiction. Therefore, $N_{\delta}<+\infty$ for all $\delta \in \mathbb{R}_{+}, N_{\delta_{1}} \leqslant N_{\delta_{2}}$ for all $\delta_{1} \leqslant \delta_{2}$ by construction, $\lim _{\delta \rightarrow+\infty} N_{\delta}=+\infty$ due to the property $\sup _{\mathbf{x} \in M}|\mathbf{x}|_{\mathcal{W}}=+\infty$, then $R=\inf _{\delta \in \mathbb{R}_{+}} N_{\delta}$ is as required and for any $\varepsilon \geqslant R$ there exists $\delta \geqslant 0$ with the property $N_{\delta}=\varepsilon$.

Let $\mathcal{W}=\mathcal{A} \cup \mathcal{R} \cup \mathcal{H}$, where the set $\mathcal{A}$ is composed by locally attracting invariant sets, the set $\mathcal{R}$ contains locally repelling invariant sets and $\mathcal{H}$ is hyperbolic invariant one (some of these sets may be empty). From now on a such set $\mathcal{W}$ constitutes the main object of investigation.

If the sets $\mathcal{R}$ and $\mathcal{H}$ are not empty then always $R>0\left(n_{\mathbf{x}_{0}}>0\right.$ for any $\delta \rightarrow 0$ with $\left|\mathbf{x}_{0}\right|_{\mathcal{R}} \leqslant \delta$ or $\left|\mathbf{x}_{0}\right|_{\mathcal{H}} \leqslant \delta, \mathbf{x}_{0} \notin \mathcal{R} \cup \mathcal{H}$ ). If we exclude from the admissible set of initial conditions some neighborhoods of the sets $\mathcal{R}$ and $\mathcal{H}$, then it is possible to prove existence of the finite time $T_{r, \varepsilon}=\sup _{\left|\mathbf{x}_{0}\right| \mathcal{W}<r} T_{\mathbf{x}_{0}, \varepsilon}$. Let

$$
\mathcal{U}=\mathcal{R} \cup \mathcal{H}^{+}
$$

be the set containing all repulsing invariant sets of the system (3). Formally, this set is not minimal ( $\mathcal{R} \cup \mathcal{H}$ is the minimal one) and not necessarily compact due to $\mathcal{H}^{+}$ inclusion, $\mathcal{H} \subset \mathcal{U}$. According to definitions of the sets $\mathcal{A}, \mathcal{R}$ and $\mathcal{H}$, all trajectories of the system (3) with initial conditions outside of the set $\mathcal{U}$, e.g. $\mathbf{x}_{0} \in M \backslash \mathcal{U}$, asymptotically converge to the set $\mathcal{A}$. See the qualitative illustration of this claim in Fig. 4, where the set $\mathcal{X} \subset M$ satisfies the properties $\mathcal{U} \subset \mathcal{X}$ and $\mathcal{X} \cap \mathcal{A}=\emptyset$, and all the trajectories initiated outside of the set $\mathcal{X}$ converge to $\mathcal{A}$. In other words the set $\mathcal{A}$ is "globally" asymptotically stable in $M \backslash \mathcal{X}$. 
Fact 1. For any open set $\mathcal{X} \subset M, \mathcal{U} \subset \mathcal{X}, \mathcal{X} \cap \mathcal{A}=\emptyset$ and any $\varepsilon>0, r>0$ there exists $0 \leqslant T_{r, \varepsilon}<+\infty$ such that $\left|\mathbf{x}\left(t, \mathbf{x}_{0}\right)\right|_{\mathcal{W}}<\varepsilon$ for all $t \geqslant T_{r, \varepsilon}$ whenever $\mathbf{x}_{0} \in D_{r} \backslash \mathcal{X}, D_{r}=\left\{\mathbf{x} \in M:|\mathbf{x}|_{\mathcal{W}}<r\right\}$.

Proof. Since the system (3) is $\mathcal{W}$-attracting, then for each $\mathbf{x}_{0} \in M$ there exists $0 \leqslant T_{\mathbf{x}_{0}, \varepsilon}<+\infty$. Consider $T_{r, \varepsilon}=\sup _{\mathbf{x}_{0} \in D_{r} \backslash \mathcal{X}} T_{\mathbf{x}_{0}, \varepsilon}$ and assume by contradiction that $T_{r, \varepsilon}=+\infty$ for some $r>0, \varepsilon>0$. Then there exists some sequence of $\mathbf{x}_{i} \in D_{r} \backslash \mathcal{X}, i \geqslant 0$ such that the corresponding $T_{\mathbf{x}_{i}, \varepsilon} \rightarrow+\infty$ as $\mathbf{x}_{i} \rightarrow \mathbf{x}_{\infty}$, where $\mathbf{x}_{\infty}$ belongs to a boundary of the sets $D_{r}$ and $\mathcal{X}$. Consider the initial conditions $\mathbf{x}_{j} \in \mathcal{X}$ or $\mathbf{x}_{j} \in M \backslash D_{r}$ for which $\mathbf{x}\left(t^{\prime}, \mathbf{x}_{j}\right)=\mathbf{x}_{\infty}$ for some $t^{\prime}>0$ (since the set $D_{r} \backslash \mathcal{X}$ contains all the attracting invariants, such trajectories should exist). Clearly $T_{\mathbf{x}_{j}, \varepsilon}=+\infty$ for these initial conditions that is a contradiction, since it implies that the trajectory $\mathbf{x}\left(t, \mathbf{x}_{\infty}\right)$ has its own invariant set outside of $\mathcal{W}$.

3.4. $\mathcal{K} \mathcal{L}$ estimates. The next interesting question is the existence of $\mathcal{K} \mathcal{L}$ estimates for the solutions of a $\mathcal{W}$-asymptotically stable system. If we exclude the neighborhoods of the set $\mathcal{U}$, then existence of the required estimate can be proven applying the standard arguments [16].

Fact 2. For any open neighborhood $\mathcal{X} \subset M, \mathcal{U} \subset \mathcal{X}, \mathcal{X} \cap \mathcal{A}=\emptyset$ there exists $\beta \in \mathcal{K} \mathcal{L}$ such that

$$
\left|\mathbf{x}\left(t, \mathbf{x}_{0}\right)\right|_{\mathcal{W}} \leqslant \beta\left(\left|\mathbf{x}_{0}\right|_{\mathcal{W}}+R, t\right), t \geqslant 0
$$

for all $\mathbf{x}_{0} \in M \backslash \mathcal{X}$.

Proof. From proposition 1 there exists a function $\phi \in \mathcal{K}$ such that $\left|\mathbf{x}\left(t, \mathbf{x}_{0}\right)\right|_{\mathcal{W}} \leqslant$ $\phi\left(\left|\mathbf{x}_{0}\right|_{\mathcal{W}}+R\right), t \geqslant 0$ for all $\mathbf{x}_{0} \in M, R>0$. From fact 1 , for all $\varepsilon>0, r>0$ there exists $0 \leqslant T_{r, \varepsilon}<+\infty$ such that $\left|\mathbf{x}\left(t, \mathbf{x}_{0}\right)\right|_{\mathcal{W}}<\varepsilon$ for all $t \geqslant T_{r, \varepsilon}$ whenever $\mathbf{x}_{0} \in D_{r} \backslash \mathcal{X}$. Applying the same arguments from the proof of Lemma 3.1 [16], we prove the existence of the map $T: \mathbb{R}_{>0} \times \mathbb{R}_{>0} \rightarrow \mathbb{R}_{>0}$ such that: $T(r, \cdot)$ is continuous and strictly decreasing for each fixed $r>0 ; T(\cdot, s)$ is increasing for $s>0, \lim _{r \rightarrow+\infty} T(r, s)=+\infty$; and $\left|\mathbf{x}\left(t, \mathbf{x}_{0}\right)\right|_{\mathcal{W}}<\varepsilon$ whenever $\mathbf{x}_{0} \in D_{r} \backslash \mathcal{X}$ for all $t \geqslant T(r, \varepsilon)$. Further, as in the proof of Proposition 2.5 [16], if we define $\psi(r, \cdot)=$ $T^{-1}(r, \cdot)$, then for all $r>0$ the map $\psi(r, \cdot)$ is continuous and strictly decreasing, $\psi(r, 0)=+\infty$. By definition $\left|\mathbf{x}\left(t, \mathbf{x}_{0}\right)\right|_{\mathcal{W}}<\psi(r, t) \leqslant \psi(r+R, t)$ whenever $\mathbf{x}_{0} \in$ $D_{r} \backslash \mathcal{X}$ for all $t \geqslant 0$ and

$$
\left|\mathbf{x}\left(t, \mathbf{x}_{0}\right)\right|_{\mathcal{W}}<\tilde{\psi}\left(\left|\mathbf{x}_{0}\right|_{\mathcal{W}}+R, t\right), t \geqslant 0, \mathbf{x}_{0} \in M \backslash \mathcal{X}
$$

where $\tilde{\psi}(s, t)=\min \left\{\phi(s), \inf _{r>s} \psi(r, t)\right\}$. If the function $\tilde{\psi}$ is not from the class $\mathcal{K} \mathcal{L}$, then it can be upper bounded by a function $\beta \in \mathcal{K} \mathcal{L}$ with the property $\tilde{\psi}(s, t) \leqslant$ $\beta(s, t)$ for any $s \in \mathbb{R}_{+}, t \in \mathbb{R}_{+}[16]$.

For $\mathbf{x}_{0} \in M \backslash \mathcal{X}$, there is an open set $\mathcal{X}^{\prime} \subset \mathcal{X}, \mathcal{U} \subset \mathcal{X}^{\prime}$ such that $\mathbf{x}\left(t, \mathbf{x}_{0}\right) \in M \backslash \mathcal{X}^{\prime}$ for all $t \geqslant 0$. In this set, all trajectories converge to "globally" asymptotically stable set $\mathcal{A}$, and this is a conventional case (see Fig. 4). That explains why the reasoning from [16] is valid to prove fact 2 .

Let $\mathcal{X}=B_{\rho}$ for some $\rho>0, B_{\rho}=\left\{\mathbf{x} \in M:|\mathbf{x}|_{\mathcal{U}}<\rho\right\}$. Clearly, there exists $\rho_{\max }>0$ such that the properties $\mathcal{U} \subset B_{\rho}, B_{\rho} \cap \mathcal{A}=\emptyset$ hold only for $\rho \leqslant \rho_{\max }$ (if $\rho>\rho_{\max }$, then $B_{\rho} \cap \mathcal{A} \neq \emptyset$ due to compactness of $\mathcal{W}$ ). We also restrict the value of $\rho_{\max }$ assuming that the quantity $|\mathbf{x}|_{\mathcal{R} \cup \mathcal{H}} \leqslant \rho_{\max }$ implies $|\mathbf{x}|_{\mathcal{W}}=|\mathbf{x}|_{\mathcal{R}}$ or $|\mathbf{x}|_{\mathcal{W}}=|\mathbf{x}|_{\mathcal{H}}$ 
Fact 3. For any $0<\rho \leqslant \rho_{\max }$ there exist $\beta \in \mathcal{K} \mathcal{L}, \eta \in \mathcal{K}$ such that

$$
\left|\mathbf{x}\left(t, \mathbf{x}_{0}\right)\right|_{\mathcal{W}} \leqslant \beta\left(\left|\mathbf{x}_{0}\right|_{\mathcal{W}}+R, \eta(\rho) t\right)
$$

for all $\mathbf{x}_{0} \in M \backslash B_{\rho}$.

Proof. From fact 2 , there exists $\beta \in \mathcal{K} \mathcal{L}$ such that $\left|\mathbf{x}\left(t, \mathbf{x}_{0}\right)\right|_{\mathcal{W}} \leqslant \beta\left(\left|\mathbf{x}_{0}\right|_{\mathcal{W}}+R, t\right)$, $t \geqslant 0$ for all $\mathbf{x}_{0} \in M \backslash B_{\rho_{\max }}$, and from proposition 1 , there exists a function $\phi \in \mathcal{K}$ such that $\left|\mathbf{x}\left(t, \mathbf{x}_{0}\right)\right| \mathcal{W} \leqslant \phi\left(\left|\mathbf{x}_{0}\right| \mathcal{W}+R\right), t \geqslant 0$ for all $\mathbf{x}_{0} \in M, R \geqslant 0$. Owning the result from [28], there exist functions $\sigma_{1}, \sigma_{2} \in \mathcal{K}_{\infty}$ such that

$$
\beta(s, t) \leqslant \sigma_{1}(s) \sigma_{2}\left(e^{-t}\right)
$$

for all $s \in \mathbb{R}_{+}, t \in \mathbb{R}_{+}$.

For any $0<\rho \leqslant \rho_{\max }$ and $r \in \mathbb{R}_{+}$define the sets $\mathcal{L}_{\rho}=\left\{\mathbf{x} \in M: \rho \leqslant|\mathbf{x}|_{\mathcal{U}} \leqslant\right.$ $\left.\rho_{\max }\right\}$ and $\mathcal{E}_{r}=\left\{\mathbf{x} \in M:|\mathbf{x}|_{\mathcal{W}} \leqslant r\right\}$. Then for any $0<\rho \leqslant \rho_{\max }$ and $r \in \mathbb{R}_{+}$for all $\mathbf{x}_{0} \in \mathcal{L}_{\rho}$ we can define

$$
\begin{gathered}
\tau_{\mathbf{x}_{0}}= \begin{cases}\arg \inf _{t \geqslant 0}\left\{\mathbf{x}\left(t, \mathbf{x}_{0}\right) \in M \backslash B_{\rho_{\max }}\right\} & \text { if } \mathbf{x}_{0} \notin \mathcal{U} ; \\
+\infty & \text { if } \mathbf{x}_{0} \in \mathcal{U},\end{cases} \\
\bar{\tau}(r, \rho)=\sup _{\mathbf{x}_{0} \in \mathcal{E}_{r} \cap \mathcal{L}_{\rho}} \tau_{\mathbf{x}_{0} .} .
\end{gathered}
$$

The time instants $\tau_{\mathbf{x}_{0}}$ are well defined for all $\mathbf{x}_{0} \notin \mathcal{U}$ (all trajectories with initial conditions in $B_{\rho_{\max }} \backslash \mathcal{U}$ enter in a finite time the set $\left.M \backslash B_{\rho_{\max }}\right)$, and for initial conditions $\mathbf{x}_{0} \in \mathcal{U}$ the value $\tau_{\mathbf{x}_{0}}$ is infinite. Hence, for the trajectories initiated into the compact set $\mathcal{E}_{r} \cap \mathcal{L}_{\rho}$ the value $\bar{\tau}(r, \rho)$ estimates the maximal time of the set $M \backslash B_{\rho_{\max }}$ access, it is well defined for any $r \in \mathbb{R}_{+}$and $0<\rho \leqslant \rho_{\max }$ (if the intersection $\mathcal{E}_{r} \cap \mathcal{L}_{\rho}$ is empty we put $\left.\bar{\tau}(r, \rho)=0\right)$. For any fixed $\rho \in\left(0, \rho_{\max }\right]$ the function $\bar{\tau}(\cdot, \rho)$ is non-decreasing, for any $r \in \mathbb{R}_{+}$the function $\bar{\tau}(r, \cdot)$ is non-increasing $\left(\lim _{\rho \rightarrow 0} \bar{\tau}(r, \rho)=+\infty, \lim _{\rho \rightarrow \rho_{\max }} \bar{\tau}(r, \rho)=0\right)$. The function $\bar{\tau}(r, \rho)$ equals to zero for all $\rho \geqslant \rho_{\max }$ and $r<\rho$ (if the intersection $\mathcal{E}_{r} \cap \mathcal{L}_{\rho}$ is empty), in the domain $\mathcal{P}=\left\{(r, \rho): r \geqslant \rho\right.$ and $\left.\rho \leqslant \rho_{\max }\right\}$ the function $\bar{\tau}(r, \rho)$ inherits continuity after the system solutions. Then for all $0<\rho \leqslant \rho_{\max }$ and $r \in \mathbb{R}_{+}$there exist $\varrho \in \mathcal{K}_{\infty}, \eta \in \mathcal{K}$ with $\eta\left(\rho_{\max }\right)=1$ such that

$$
\bar{\tau}(r, \rho) \leqslant \varrho(r) / \eta(\rho) .
$$

Indeed, define $\tilde{\rho}=1 / \rho$ and $\tilde{\tau}(r, \tilde{\rho})=\bar{\tau}(r, 1 / \tilde{\rho})$, then $\tilde{\tau}$ is a non-decreasing function of both arguments $r \in \mathbb{R}_{+}, \tilde{\rho} \in\left[1 / \rho_{\max },+\infty\right)$ and using the result of [28] in the domain $\mathcal{P}$ we can find some $\tilde{\varrho} \in \mathcal{K}_{\infty}, \tilde{\eta} \in \mathcal{K}_{\infty}$ such that $\tilde{\tau}(r, \tilde{\rho}) \leqslant \tilde{\varrho}(r) \tilde{\eta}(\tilde{\rho})$ for all $(r, \tilde{\rho}) \in \mathcal{P}$. Since outside $\mathcal{P}$ the function $\tilde{\tau}$ is zero and $\tilde{\varrho}(r) \tilde{\eta}(\tilde{\rho})$ stay nonnegative everywhere for $r \in \mathbb{R}_{+}, \tilde{\rho} \in \mathbb{R}_{+}$, then the inequality $\tilde{\tau}(r, \tilde{\rho}) \leqslant \tilde{\varrho}(r) \tilde{\eta}(\tilde{\rho})$ is valid for all $r \in \mathbb{R}_{+}, \tilde{\rho} \in\left[1 / \rho_{\max },+\infty\right)$. The existence of functions $\varrho \in \mathcal{K}_{\infty}, \eta \in \mathcal{K}$ follows returning to $\rho=1 / \tilde{\rho}$ (under a suitable rescaling in order to provide that $\left.\eta\left(\rho_{\max }\right)=1\right)$.

Note that for any $0<\rho \leqslant \rho_{\max }$ the upper estimate

$$
\left|\mathbf{x}\left(t, \mathbf{x}_{0}\right)\right|_{\mathcal{W}} \leqslant \beta\left(\left|\mathbf{x}_{0}\right|_{\mathcal{W}}+R, \eta(\rho) t\right), t \geqslant 0
$$

is satisfied for all $\mathbf{x}_{0} \in M \backslash B_{\rho_{\max }}\left(\eta\left(\rho_{\max }\right) \leqslant 1\right.$ for any $\left.0<\rho \leqslant \rho_{\max }\right)$. Let us fix a $\rho \in\left(0, \rho_{\max }\right]$ and take some $\mathbf{x}_{0} \in \mathcal{L}_{\rho}$, then we have two estimates:

$$
\left|\mathbf{x}\left(t, \mathbf{x}_{0}\right)\right|_{\mathcal{W}} \leqslant \phi\left(\left|\mathbf{x}_{0}\right|_{\mathcal{W}}+R\right)
$$


for all $t \geqslant 0$ and

$$
\left|\mathbf{x}\left(t, \mathbf{x}_{0}\right)\right|_{\mathcal{W}} \leqslant \beta\left[\left|\mathbf{x}\left(\tau_{\mathbf{x}_{0}}, \mathbf{x}_{0}\right)\right|_{\mathcal{W}}+R, \eta(\rho)\left(t-\tau_{\mathbf{x}_{0}}\right)\right]
$$

for all $t \geqslant \tau_{\mathbf{x}_{0}}$. Substituting the first estimate into the second one we get

$$
\begin{aligned}
\left|\mathbf{x}\left(t, \mathbf{x}_{0}\right)\right|_{\mathcal{W}} & \leqslant \beta\left[\phi\left(\left|\mathbf{x}_{0}\right| \mathcal{W}+R\right)+R, \eta(\rho)\left(t-\tau_{\mathbf{x}_{0}}\right)\right] \\
& \leqslant \sigma_{1}\left[\phi\left(\left|\mathbf{x}_{0}\right| \mathcal{W}+R\right)+R\right] \sigma_{2}\left(e^{-\eta(\rho)\left(t-\tau_{\mathbf{x}_{0}}\right)}\right) \\
& \leqslant \sigma_{1}\left[\phi\left(\left|\mathbf{x}_{0}\right| \mathcal{W}+R\right)+R\right] \sigma_{2}\left(e^{-\eta(\rho) t} e^{\eta(\rho) \tau_{\mathbf{x}_{0}}}\right) .
\end{aligned}
$$

Since $\sigma_{1}[\phi(s+R)+R] \leqslant \sigma_{1}[2 \max \{\phi(s+R), R\}] \leqslant \sigma_{1}[2 \max \{\phi(s+R), R+s\}]$ for all $s \in \mathbb{R}_{+}$, define $\theta(s)=\max \left\{\phi(s), \sigma_{1}[2 \max \{\phi(s), s\}]\right\}$, then

$$
\left|\mathbf{x}\left(t, \mathbf{x}_{0}\right)\right|_{\mathcal{W}} \leqslant \theta\left(\left|\mathbf{x}_{0}\right| \mathcal{W}+R\right) \sigma_{2}\left(e^{\eta(\rho) \tau_{\mathbf{x}_{0}}} e^{-\eta(\rho) t}\right)
$$

for all $t \geqslant \tau_{\mathbf{x}_{0}}$. Note that $\tau_{\mathbf{x}_{0}} \leqslant \bar{\tau}\left(\left|\mathbf{x}_{0}\right| \mathcal{W}, \rho\right) \leqslant \bar{\tau}\left(\left|\mathbf{x}_{0}\right|_{\mathcal{W}}+R, \rho\right) \leqslant \varrho\left(\left|\mathbf{x}_{0}\right|_{\mathcal{W}}+R\right) / \eta(\rho)$, then

$$
\left|\mathbf{x}\left(t, \mathbf{x}_{0}\right)\right|_{\mathcal{W}} \leqslant \theta\left(\left|\mathbf{x}_{0}\right| \mathcal{W}+R\right) \sigma_{2}\left(e^{\varrho\left(\left|\mathbf{x}_{0}\right| \mathcal{W}+R\right)} e^{-\eta(\rho) t}\right)
$$

for all $t \geqslant \tau_{\mathbf{x}_{0}}$. Define $\tilde{\beta}(s, t)=\max \left\{1,1 / \sigma_{2}(1)\right\} \theta(s) \sigma_{2}\left(e^{\varrho(s)} e^{-t}\right)$, obviously $\tilde{\beta} \in \mathcal{K} \mathcal{L}$, then for any $\mathbf{x}_{0} \in \mathcal{L}_{\rho}$ the estimate is satisfied:

$$
\left|\mathbf{x}\left(t, \mathbf{x}_{0}\right)\right|_{\mathcal{W}} \leqslant \tilde{\beta}\left(\left|\mathbf{x}_{0}\right|_{\mathcal{W}}+R, \eta(\rho) t\right)
$$

for all $t \geqslant 0$. Indeed, the estimate (4) holds for all $t \geqslant \tau_{\mathbf{x}_{0}}$ by construction and the consideration above. For $t=\tau_{\mathbf{x}_{0}}$ we have

$$
\begin{aligned}
\theta\left(\left|\mathbf{x}_{0}\right| \mathcal{W}+R\right) \sigma_{2}\left(e^{\varrho\left(\left|\mathbf{x}_{0}\right| \mathcal{W}+R\right)} e^{-\eta(\rho) \tau_{\mathbf{x}_{0}}}\right) & =\theta\left(\left|\mathbf{x}_{0}\right| \mathcal{W}+R\right) \sigma_{2}\left(e^{\varrho\left(\left|\mathbf{x}_{0}\right| \mathcal{W}+R\right)-\eta(\rho) \tau_{\mathbf{x}_{0}}}\right) \\
& \geqslant \theta\left(\left|\mathbf{x}_{0}\right| \mathcal{W}+R\right) \sigma_{2}(1) \geqslant \phi\left(\left|\mathbf{x}_{0}\right| \mathcal{W}+R\right) \sigma_{2}(1),
\end{aligned}
$$

therefore $\tilde{\beta}\left(\left|\mathbf{x}_{0}\right| \mathcal{W}+R, \eta(\rho) \tau_{\mathbf{x}_{0}}\right) \geqslant \phi\left(\left|\mathbf{x}_{0}\right| \mathcal{W}+R\right)$ and the estimate (4) is also valid for all $t \in\left[0, \tau_{\mathbf{x}_{0}}\right]$. Next, for any $\mathbf{x}_{0} \in M \backslash B_{\rho_{\max }}$ the estimate $\left|\mathbf{x}\left(t, \mathbf{x}_{0}\right)\right|_{\mathcal{W}} \leqslant \beta\left(\left|\mathbf{x}_{0}\right| \mathcal{W}+\right.$ $R, t)$ is satisfied for all $t \geqslant 0$, since $\beta(s, t) \leq \tilde{\beta}(s, t)$ for all $s \in \mathbb{R}_{+}, t \in \mathbb{R}_{+}$, then the estimate (4) is valid for all $\mathbf{x}_{0} \in M \backslash B_{\rho_{\max }}$ and $t \geqslant 0$. Therefore, for any $0<\rho \leqslant \rho_{\max }$ there exist functions $\tilde{\beta} \in \mathcal{K} \mathcal{L}, \eta \in \mathcal{K}$ such that for all $\mathbf{x}_{0} \in M \backslash B_{\rho}$,

$$
\left|\mathbf{x}\left(t, \mathbf{x}_{0}\right)\right|_{\mathcal{W}} \leqslant \tilde{\beta}\left(\left|\mathbf{x}_{0}\right|_{\mathcal{W}}+R, \eta(\rho) t\right), t \geqslant 0 .
$$

Now we are in position to propose $\mathcal{K} \mathcal{L}$ estimates for the solutions of $\mathcal{W}$-asymptotically stable system (3).

Proposition 2. For the system (3) the following is equivalent:

$-\mathcal{W}$-asymptotic stability, $\mathcal{W}=\mathcal{A} \cup \mathcal{R} \cup \mathcal{H}$;

- for all $\mathbf{x}_{0} \in M$ there exist functions $\beta \in \mathcal{K} \mathcal{L}, \eta \in \mathcal{K}$ such that

$$
\left|\mathbf{x}\left(t, \mathbf{x}_{0}\right)\right|_{\mathcal{W}} \leqslant \beta\left(\left|\mathbf{x}_{0}\right|_{\mathcal{W}}+R, \min \left\{1, \eta\left(\left|\mathbf{x}_{0}\right|_{\mathcal{U}}\right)\right\} t\right), \mathcal{U}=\mathcal{R} \cup \mathcal{H}^{+}, t \geqslant 0 .
$$

Proof. The sufficiency is clear, $\mathcal{W}$-attractivity follows for all $\mathbf{x}_{0} \in M \backslash \mathcal{W}$ from the $\mathcal{K} \mathcal{L}$ estimate, and $\left|\mathbf{x}\left(t, \mathbf{x}_{0}\right)\right| \mathcal{W} \leqslant \phi\left(\left|\mathbf{x}_{0}\right| \mathcal{W}+R\right), t \geqslant 0, \phi(s)=\beta(s, 0)$ is equivalent to $\mathcal{W}$-stability from proposition 1 .

Necessity. The system (3) is $\mathcal{W}$-asymptotically stable, the set $\mathcal{W}$ contains all invariant solutions and $\varlimsup_{t \rightarrow+\infty}\left|\mathbf{x}\left(t, \mathbf{x}_{0}\right)\right|_{\mathcal{W}}=0$ for all $\mathbf{x}_{0} \in M$. From fact 3 , there exist $\beta \in \mathcal{K} \mathcal{L}, \eta \in \mathcal{K}$ such that

$$
\left|\mathbf{x}\left(t, \mathbf{x}_{0}\right)\right|_{\mathcal{W}} \leqslant \beta\left(\left|\mathbf{x}_{0}\right|_{\mathcal{W}}+R, \eta(\rho) t\right)
$$


for all $\mathbf{x}_{0} \in M \backslash B_{\rho}, B_{\rho}=\left\{\mathbf{x} \in M:|\mathbf{x}|_{\mathcal{U}}<\rho\right\}$ for all $0<\rho \leqslant \rho_{\max }<+\infty$, then substitution $\rho=\left|\mathbf{x}_{0}\right| \mathcal{U}\left(\eta\left(\rho_{\max }\right)=1\right)$ implies the result.

For the case $\mathbf{x}_{0} \in \mathcal{W}$, the proposed estimate is not meaningful since it can be reduced to

$$
0 \leqslant \beta\left(R, \min \left\{1, \eta\left(\left|\mathbf{x}_{0}\right| \mathcal{U}\right)\right\} t\right),
$$

which is correct but useless. If $\mathbf{x}_{0} \in \mathcal{H}^{+}$, then the worst case estimate $\left|\mathbf{x}\left(t, \mathbf{x}_{0}\right)\right|_{\mathcal{W}} \leqslant$ $\beta\left(\left|\mathbf{x}_{0}\right| \mathcal{W}+R, 0\right)$ is satisfied. In this case the upper bound on the trajectories norm from proposition 2 does not evaluate the rate of convergence to the set $\mathcal{H}$. All these shortages are caused by the sets $\mathcal{R}$ and $\mathcal{H}$ presence.

Remark 1 . The proposed $\mathcal{W}$-asymptotic stability property is not related to the stability with respect to two measures $[14,32]$ since the upper term here is not a measure (the measure has to be zero on the set of interest). In addition, if $\mathcal{U}=\emptyset$, then we have $\mathcal{W}=\mathcal{A}$ and the estimate above is reduced to $\left|\mathbf{x}\left(t, \mathbf{x}_{0}\right)\right|_{\mathcal{A}} \leqslant \beta\left(\left|\mathbf{x}_{0}\right|_{\mathcal{A}}, t\right)$, which is a conventional estimate for the globally asymptotically stable set $\mathcal{A}$ in the Lyapunov sense [16].

\section{LYAPUNOV FUNCTIONS}

This section has four parts. In the first one, Lyapunov functions for the system (3) and the set $\mathcal{W}$ are introduced and the main result is formulated. Proofs of the main result are presented in the last three subsections.

4.1. Lyapunov function definition. In this subsection for the set $\mathcal{W}$ defined as above, an equivalent characterization of $\mathcal{W}$-asymptotic stability in terms of Lyapunov functions is proposed.

Definition 2. A smooth function $W: M \rightarrow \mathbb{R}_{+}$is called $\mathcal{W}$-Lyapunov function for the system (3) if

- there exist functions $\alpha_{1}, \alpha_{2} \in \mathcal{K}_{\infty}$ and a constant $R \geqslant 0$ such that for all $\mathrm{x} \in M$,

$$
\alpha_{1}\left(|\mathbf{x}|_{\mathcal{W}}\right) \leqslant W(\mathbf{x}) \leqslant \alpha_{2}\left(|\mathbf{x}|_{\mathcal{W}}+R\right)
$$

- there exist functions $\chi, \alpha_{3} \in \mathcal{K}$ such that for all $\mathbf{x} \in M$,

$$
\begin{gathered}
L_{\mathbf{f}(\mathbf{x})} W(\mathbf{x})=\partial W(\mathbf{x}) / \partial \mathbf{x} \mathbf{f}(\mathbf{x}) \leqslant-\min \{1, \chi(|\mathbf{x}| \mathcal{U})\} \alpha_{3}(W(\mathbf{x})), \\
\mathcal{W}=\mathcal{A} \cup \mathcal{R} \cup \mathcal{H}, \mathcal{U}=\mathcal{R} \cup \mathcal{H}^{+} .
\end{gathered}
$$

For a continuous function $\mathbf{h}: M \rightarrow \mathbb{R}^{m}$ and a set $\mathcal{W}$, the system (3) is called $\mathcal{W}$-detectable with respect to the output $\mathbf{y}=\mathbf{h}(\mathbf{x})$, if for all $\mathbf{x}_{0} \in M$,

$$
\mathbf{y}(t)=\mathbf{h}\left(\mathbf{x}\left(t, \mathbf{x}_{0}\right)\right) \equiv 0, t \geqslant 0 \Longrightarrow \varlimsup_{t \rightarrow+\infty}\left|\mathbf{x}\left(t, \mathbf{x}_{0}\right)\right|_{\mathcal{W}}=0
$$

Define $\mathcal{Z}=\{\mathbf{x} \in M: \mathbf{h}(\mathbf{x})=0\}$. The detectability property implies that any trajectory located in a forward invariant subset of $\mathcal{Z}$ converges to the set $\mathcal{W}$.

Definition 3. A smooth function $W: M \rightarrow \mathbb{R}_{+}$is called weak $\mathcal{W}$-Lyapunov function for the system (3) if

- there exist functions $\alpha_{1}, \alpha_{2} \in \mathcal{K}_{\infty}$ and a constant $R \geqslant 0$ such that for all $\mathrm{x} \in M$,

$$
\alpha_{1}\left(|\mathbf{x}|_{\mathcal{W}}\right) \leqslant W(\mathbf{x}) \leqslant \alpha_{2}\left(|\mathbf{x}|_{\mathcal{W}}+R\right)
$$


- there exists a function $\kappa \in \mathcal{K}$ and a continuous function $\mathbf{h}: M \rightarrow \mathbb{R}^{m}$ such that for all $\mathrm{x} \in M$,

$$
L_{\mathbf{f}(\mathbf{x})} W(\mathbf{x}) \leqslant-\kappa(|\mathbf{h}(\mathbf{x})|)
$$

and the system $(3)$ is $\mathcal{W}$-detectable with respect to the output $\mathbf{y}=\mathbf{h}(\mathbf{x})$.

Note that in motivating examples the weak $\mathcal{W}$-Lyapunov functions have been observed. Relations between these Lyapunov functions are the main result of the paper.

Theorem 1. For the system (3) and a compact set $\mathcal{W}$, the existence of a weak $\mathcal{W}$-Lyapunov function implies $\mathcal{W}$-asymptotic stability.

Theorem 2. For the system (3) and the compact set $\mathcal{W}=\mathcal{A} \cup \mathcal{R} \cup \mathcal{H}$, where the sets $\mathcal{A}, \mathcal{R}$ and $\mathcal{H}$ are composed by locally attracting, repelling and hyperbolic invariant subsets respectively, the following is equivalent:

1) $\mathcal{W}$-asymptotic stability;

2) existence of a $\mathcal{W}$-Lyapunov function;

3) existence of a weak $\mathcal{W}$-Lyapunov function.

Since the existence of weak $\mathcal{W}$-Lyapunov functions is established in the motivating examples, these systems also possess Lyapunov functions and $\mathcal{K} \mathcal{L}$ estimates (theorem 2, proposition 2). Existence of such Lyapunov functions implies a kind of stability robustness of the system with respect to external disturbances or parametric uncertainties. Other examples of such systems are presented in section 5.

4.2. Proof of theorem 1. Under conditions of theorem 1, for the system (3), there exists a smooth function $W: M \rightarrow \mathbb{R}_{+}$such that for all $\mathbf{x} \in M$

$$
\begin{gathered}
\alpha_{1}\left(|\mathbf{x}|_{\mathcal{W}}\right) \leqslant W(\mathbf{x}) \leqslant \alpha_{2}\left(|\mathbf{x}|_{\mathcal{W}}+R\right), \alpha_{1}, \alpha_{2} \in \mathcal{K}_{\infty}, R \geqslant 0 \\
L_{\mathbf{f}(\mathbf{x})} W(\mathbf{x}) \leqslant-\kappa(|\mathbf{h}(\mathbf{x})|), \kappa \in \mathcal{K}
\end{gathered}
$$

for some continuous function $\mathbf{h}: M \rightarrow \mathbb{R}^{m}$, and the system (3) is $\mathcal{W}$-detectable with respect to the output $\mathbf{y}=\mathbf{h}(\mathbf{x})$. Since $\dot{W} \leqslant 0$, then $W(t)$ is bounded and $\alpha_{1}(|\mathbf{x}(t)| \mathcal{W}) \leqslant W(\mathbf{x}(t)) \leqslant W(\mathbf{x}(0)) \leqslant \alpha_{2}(|\mathbf{x}(0)| \mathcal{W}+R)$ for all $t \geqslant 0$. This inequality implies that $|\mathbf{x}(t)| \mathcal{W} \leqslant \phi(|\mathbf{x}(0)| \mathcal{W}+R), \phi(s)=\alpha_{1}^{-1} \circ \alpha_{2}(s)$. According to proposition 1 this is equivalent to $\mathcal{W}$-stability. Due to compactness of the set $\mathcal{W}$, there exists a function $\mu_{2} \in \mathcal{K}$ and a constant $D \in \mathbb{R}_{+}$such that $|\mathbf{x}(t)| \leqslant \mu_{2}\left(|\mathbf{x}(t)|_{\mathcal{W}}+D\right)$ and the state $\mathbf{x}(t)$ is bounded (any solution is defined for all $t \geqslant 0$ ). Thus there exists a forward invariant attracting compact set $\Omega(\mathbf{x}(0))$ for each trajectory. If we take $\mathbf{x}_{0} \in \Omega(\mathbf{x}(0))$, then $\mathbf{x}\left(t, \mathbf{x}_{0}\right) \in \Omega(\mathbf{x}(0)), t \geqslant 0$ and $W\left(\mathbf{x}\left(t, \mathbf{x}_{0}\right)\right)=c, t \geqslant 0$ for some $c \in \mathbb{R}_{+}$due to positive definiteness of $W$ and negative semi-definiteness of $\dot{W}$. This implies that $\dot{W}\left(\mathbf{x}\left(t, \mathbf{x}_{0}\right)\right)=0, t \geqslant 0$, and for $\kappa \in \mathcal{K}$ this is equivalent to $\mathbf{y}(t)=\mathbf{h}\left(\mathbf{x}\left(t, \mathbf{x}_{0}\right)\right) \equiv 0, t \geqslant 0$. Since the system is $\mathcal{W}$-detectable with respect to the output $\mathbf{y}(t)$, the equality $\varlimsup_{t \rightarrow+\infty}\left|\mathbf{x}\left(t, \mathbf{x}_{0}\right)\right| \mathcal{W}=0$ holds and the system (3) is $\mathcal{W}$-attracting.

4.3. Proof of theorem 2. The theorem is proven as follows: (1) $\Rightarrow(2) \Rightarrow(3) \Rightarrow(1)$. The link $(3) \Rightarrow(1)$ has been substantiated in theorem 1 . 
Proof $(2) \Rightarrow(3)$. Under conditions of theorem 2 , for the system (3), there exists a smooth function $W: M \rightarrow \mathbb{R}_{+}$such that for all $\mathbf{x} \in M$

$$
\begin{gathered}
\alpha_{1}\left(|\mathbf{x}|_{\mathcal{W}}\right) \leqslant W(\mathbf{x}) \leqslant \alpha_{2}\left(|\mathbf{x}|_{\mathcal{W}}+R\right), \alpha_{1}, \alpha_{2} \in \mathcal{K}_{\infty}, R \geqslant 0 \\
L_{\mathbf{f}(\mathbf{x})} W(\mathbf{x}) \leqslant-\min \left\{1, \chi\left(|\mathbf{x}|_{\mathcal{U}}\right)\right\} \alpha_{3}(W(\mathbf{x})), \chi, \alpha_{3} \in \mathcal{K} .
\end{gathered}
$$

If we denote $h(\mathbf{x})=\min \left\{1, \chi\left(|\mathbf{x}|_{\mathcal{U}}\right)\right\} \alpha_{3}(W(\mathbf{x}))$, then $h: M \rightarrow \mathbb{R}_{+}$is continuous and $W$ is a weak Lyapunov function since the system $(3)$ is $\mathcal{W}$-detectable with respect to the output $y=h(\mathbf{x})$. Next, the result follows theorem 1 .

The proof $(2) \Rightarrow(1)$ (this part of the proof is presented for completeness) relies on the fact that for any $\mathbf{x}_{0} \in M$ such that $\chi\left(\left|\mathbf{x}_{0}\right| \mathcal{U}\right)<1$, the inequality $\left|\mathbf{x}\left(t, \mathbf{x}_{0}\right)\right|_{\mathcal{U}} \geqslant$ $\nu\left(\left|\mathbf{x}_{0}\right| \mathcal{U}\right)$ holds for all $t \geqslant 0$ and for some function $\nu \in \mathcal{K}$ (the set $\mathcal{U}$ contains locally repelling subsets only, for instance, $\left.\nu(s)=s /(s+1) \inf _{\left|\mathbf{x}_{0}\right| \mathcal{U}=s, t \geqslant 0}\left|\mathbf{x}\left(t, \mathbf{x}_{0}\right)\right|_{\mathcal{U}}\right)$. Consequently

$$
L_{\mathbf{f}(\mathbf{x})} W(\mathbf{x}) \leqslant-\min \{1, \chi(|\mathbf{x}| \mathcal{U})\} \alpha_{3}(W(\mathbf{x})) \leqslant-\min \left\{1, \eta\left(\left|\mathbf{x}_{0}\right| \mathcal{U}\right)\right\} \alpha_{3}(W(\mathbf{x})),
$$

$\eta(s)=\chi \circ \nu(s)$. Using standard arguments (see Lemma 4.4 in [16]) this implies the estimate

$$
\left|\mathbf{x}\left(t, \mathbf{x}_{0}\right)\right|_{\mathcal{W}} \leqslant \beta\left(\left|\mathbf{x}_{0}\right|_{\mathcal{W}}+R, \min \left\{1, \eta\left(\left|\mathbf{x}_{0}\right| \mathcal{U}\right)\right\} t\right), t \geqslant 0, \beta \in \mathcal{K} \mathcal{L}
$$

which is equivalent to $\mathcal{W}$-asymptotic stability from proposition 2 .

Proof $(1) \Rightarrow(2)$. In this case, for all $\mathbf{x}_{0} \in M$ there exist $\phi \in \mathcal{K}$ and $R \geqslant 0$ such that $\left|\mathbf{x}\left(t, \mathbf{x}_{0}\right)\right|_{\mathcal{W}} \leqslant \phi\left(\left|\mathbf{x}_{0}\right|_{\mathcal{W}}+R\right), t \geqslant 0$ (the $\mathcal{W}$-stability equivalent definition from proposition 1 ), and for any $\varepsilon>0$ there exists $0 \leqslant T_{\mathbf{x}_{0}, \varepsilon}<+\infty$ such that $\left|\mathbf{x}\left(t, \mathbf{x}_{0}\right)\right|_{\mathcal{W}}<\varepsilon$ for all $t \geqslant T_{\mathbf{x}_{0}, \varepsilon}$. The set $\mathcal{W}=\mathcal{A} \cup \mathcal{R} \cup \mathcal{H}$ and $\mathcal{U}=\mathcal{R} \cup \mathcal{H}^{+}$.

We are going to show the existence of constants $0<\rho_{1}<\rho_{2}<\rho_{\max }\left(\rho_{\max }\right.$ is the same as in fact 3) and two locally Lipschitz continuous functions $V_{1}, V_{2}: M \rightarrow \mathbb{R}_{+}$ such that

$$
\begin{gathered}
\alpha_{1}\left(|\mathbf{x}|_{\mathcal{W}}\right) \leqslant V_{1}(\mathbf{x}) \leqslant \alpha_{2}\left(|\mathbf{x}|_{\mathcal{W}}+R\right), \alpha_{1}, \alpha_{2} \in \mathcal{K}_{\infty}, \\
L_{\mathbf{f}(\mathbf{x})} V_{1}(\mathbf{x}) \leqslant-\alpha_{3}\left(V_{1}(\mathbf{x})\right), \alpha_{3} \in \mathcal{K} \text { for a.e. }|\mathbf{x}|_{\mathcal{U}} \geqslant \rho_{1} ;
\end{gathered}
$$

$0<\gamma<V_{2}(\mathbf{x})<+\infty, L_{\mathbf{f}(\mathbf{x})} V_{2}(\mathbf{x}) \leqslant-\kappa(|\mathbf{x}| \mathcal{U}) \alpha_{4}\left(V_{2}(\mathbf{x})\right), \kappa, \alpha_{4} \in \mathcal{K}$ for a.e. $|\mathbf{x}|_{\mathcal{U}} \leqslant \rho_{2}$. The existence of such functions is proven in lemmas 3 and 5 below. Applying standard partition of unity technique one can substantiate existence of a locally Lipschitz continuous function $V: M \rightarrow \mathbb{R}_{+}$such that for a.e. $\mathbf{x} \in M$ :

$$
\begin{gathered}
\tilde{\alpha}_{1}\left(|\mathbf{x}|_{\mathcal{W}}\right) \leqslant V(\mathbf{x}) \leqslant \tilde{\alpha}_{2}\left(|\mathbf{x}|_{\mathcal{W}}+R\right), \\
L_{\mathbf{f}(\mathbf{x})} V(\mathbf{x}) \leqslant-\min \left\{1, \tilde{\kappa}\left(|\mathbf{x}|_{\mathcal{U}}\right)\right\} \tilde{\alpha}_{3}(V(\mathbf{x})), \tilde{\alpha}_{1}, \tilde{\alpha}_{2} \in \mathcal{K}_{\infty}, \tilde{\alpha}_{3}, \tilde{\kappa} \in \mathcal{K} .
\end{gathered}
$$

For instance, this is the case for $V(\mathbf{x})=\lambda(\mathbf{x}) V_{1}(\mathbf{x})+(1-\lambda(\mathbf{x})) V_{2}(\mathbf{x})$ and for a sufficiently high $\gamma>0$, where $\lambda: M \rightarrow \mathbb{R}_{+}$is continuously differentiable and satisfies the following requirements:

$$
\begin{aligned}
\lambda(\mathbf{x})= & 0 \text { for all }|\mathbf{x}|_{\mathcal{U}} \leqslant \rho_{1} ; \lambda(\mathbf{x})=1 \text { for all }|\mathbf{x}|_{\mathcal{U}} \geqslant \rho_{2} ; \\
& L_{\mathbf{f}(\mathbf{x})} \lambda(\mathbf{x})>0 \text { for all } \rho_{1}<|\mathbf{x}|_{\mathcal{U}}<\rho_{2} .
\end{aligned}
$$

Existence of the function $\lambda$ follows from lemma 4 (after appropriate smoothing and scaling). From Theorem B1 in [16] there exists a smooth function $W: M \rightarrow \mathbb{R}_{+}$ such that for all $\mathrm{x} \in M$

$$
|W(\mathbf{x})-V(\mathbf{x})| \leqslant 0.5 V(\mathbf{x}), L_{\mathbf{f}(\mathbf{x})} W(\mathbf{x}) \leqslant-0.5 \min \{1, \tilde{\kappa}(|\mathbf{x}| \mathcal{U})\} \tilde{\alpha}_{3}(W(\mathbf{x})),
$$

that is necessary to prove. 
4.4. Auxiliary results. The following result is used in lemmas 3,4 .

Lemma 2. For any continuous functions $f_{1}: \mathbb{R}_{+} \rightarrow \mathbb{R}, f_{2}: \mathbb{R}_{+} \rightarrow \mathbb{R}$ and $T \in \mathbb{R}_{+}$, the following holds

$$
\begin{aligned}
& \left|\sup _{0 \leqslant t \leqslant T} f_{1}(t)-\sup _{0 \leqslant t \leqslant T} f_{2}(t)\right| \leqslant \sup _{0 \leqslant t \leqslant T}\left|f_{1}(t)-f_{2}(t)\right|, \\
& \left|\inf _{0 \leqslant t \leqslant T} f_{1}(t)-\inf _{0 \leqslant t \leqslant T} f_{2}(t)\right| \leqslant \sup _{0 \leqslant t \leqslant T}\left|f_{1}(t)-f_{2}(t)\right| .
\end{aligned}
$$

Proof. There exist $0 \leqslant t_{1} \leqslant T$ and $0 \leqslant t_{2} \leqslant T$ such that $\sup _{0 \leqslant t \leqslant T} f_{1}(t)=f_{1}\left(t_{1}\right)$ and $\sup _{0 \leqslant t \leqslant T} f_{2}(t)=f_{2}\left(t_{2}\right)$, then $\left|\sup _{0 \leqslant t \leqslant T} f_{1}(t)-\sup _{0 \leqslant t \leqslant T} f_{2}(t)\right|=\mid f_{1}\left(t_{1}\right)-$ $f_{2}\left(t_{2}\right) \mid$. For the case $t_{1}=t_{2}=t^{\prime}$ the result is obviously satisfied, and for other cases we have

(5)

$$
\begin{array}{rlrl}
f_{1}\left(t_{1}\right) \geqslant f_{2}\left(t_{2}\right) \Rightarrow\left|f_{1}\left(t_{1}\right)-f_{2}\left(t_{2}\right)\right| & = & f_{1}\left(t_{1}\right)-f_{2}\left(t_{2}\right) \\
& \leqslant f_{1}\left(t_{1}\right)-f_{2}\left(t_{1}\right)=\left|f_{1}\left(t_{1}\right)-f_{2}\left(t_{1}\right)\right|, \\
f_{1}\left(t_{1}\right)<f_{2}\left(t_{2}\right) \Rightarrow\left|f_{1}\left(t_{1}\right)-f_{2}\left(t_{2}\right)\right| & = & f_{2}\left(t_{2}\right)-f_{1}\left(t_{1}\right) \\
& \leqslant & f_{2}\left(t_{1}\right)-f_{1}\left(t_{2}\right)=\left|f_{2}\left(t_{2}\right)-f_{1}\left(t_{2}\right)\right| .
\end{array}
$$

These properties substantiate the first desired inequality.

Similarly, since $\inf _{0 \leqslant t \leqslant T} f_{1}(t)=f_{1}\left(t_{1}\right)$ and $\inf _{0 \leqslant t \leqslant T} f_{2}(t)=f_{2}\left(t_{2}\right)$ for some $0 \leqslant t_{1} \leqslant T, 0 \leqslant t_{2} \leqslant T$, then $\left|\inf _{0 \leqslant t \leqslant T} f_{1}(t)-\inf _{0 \leqslant t \leqslant T} f_{2}(t)\right|=\left|f_{1}\left(t_{1}\right)-f_{2}\left(t_{2}\right)\right|$. For the case $t_{1}=t_{2}=t^{\prime}$ the result is satisfied, and for other cases the second property follows from (5).

Recall that for $\rho_{\max }>0$ the properties $\mathcal{U} \subset B_{\rho}, B_{\rho} \cap \mathcal{A}=\emptyset$ hold only for $\rho \leqslant \rho_{\max }$.

Lemma 3. Let all conditions of theorem 2 hold. Then for any $0<\rho<\rho_{\max }$ there exists a locally Lipschitz continuous function $V_{1}: M \backslash B_{\rho} \rightarrow \mathbb{R}_{+}, B_{\rho}=\{\mathbf{x} \in M$ : $\left.|\mathbf{x}|_{\mathcal{U}}<\rho\right\}$ (continuous on the set $\mathcal{A}$ ) such that $\alpha_{1}\left(|\mathbf{x}|_{\mathcal{W}}\right) \leqslant V_{1}(\mathbf{x}) \leqslant \alpha_{2}\left(|\mathbf{x}|_{\mathcal{W}}+R\right)$, $\alpha_{1}, \alpha_{2} \in \mathcal{K}_{\infty}, L_{\mathbf{f}(\mathbf{x})} V_{1}(\mathbf{x}) \leqslant-\alpha_{3}\left(V_{1}(\mathbf{x})\right), \alpha_{3} \in \mathcal{K}$ for a.e. $|\mathbf{x}| \mathcal{U} \geqslant \rho$.

Proof. For any $\mathbf{x}_{0} \in M$, define

$$
v\left(\mathbf{x}_{0}\right)=\sup _{t \geqslant 0}\left|\mathbf{x}\left(t, \mathbf{x}_{0}\right)\right| \mathcal{W},
$$

by construction $\left|\mathbf{x}_{0}\right| \mathcal{W} \leqslant v\left(\mathbf{x}_{0}\right) \leqslant \phi\left(\left|\mathbf{x}_{0}\right| \mathcal{W}+R\right)$ and $v(\mathbf{x})=0$ for $\mathbf{x} \in \mathcal{W}$. From $\mathcal{W}$ attractivity for any $\mathbf{x}_{0} \in M$ there exists $T_{\mathbf{x}_{0}} \in \mathbb{R}_{+}$such that $v\left(\mathbf{x}_{0}\right)=\sup _{0 \leqslant t \leqslant T_{\mathbf{x}_{0}}}\left|\mathbf{x}\left(t, \mathbf{x}_{0}\right)\right|_{\mathcal{W}}$. Indeed, $T_{\mathbf{x}_{0}}=T_{\mathbf{x}_{0},\left|\mathbf{x}_{0}\right| \mathcal{W}}$ for any $\mathbf{x}_{0} \in M \backslash \mathcal{W}$ and the choice $T_{\mathbf{x}_{0}}=0$ is admissible for $\mathbf{x}_{0} \in \mathcal{W}$. To analyze continuity property of the function $v$, consider

$$
\begin{aligned}
\left|v\left(\mathbf{x}_{1}\right)-v\left(\mathbf{x}_{2}\right)\right| & =\left|\sup _{t \geqslant 0}\right| \mathbf{x}\left(t, \mathbf{x}_{1}\right)\left|\mathcal{W}-\sup _{t \geqslant 0}\right| \mathbf{x}\left(t, \mathbf{x}_{2}\right)|\mathcal{W}| \\
& =\left|\sup _{0 \leqslant t \leqslant T_{\mathbf{x}_{1}}}\right| \mathbf{x}\left(t, \mathbf{x}_{1}\right)\left|\mathcal{W}-\sup _{0 \leqslant t \leqslant T_{\mathbf{x} 2}}\right| \mathbf{x}\left(t, \mathbf{x}_{2}\right)|\mathcal{W}| \\
& \leqslant\left.\sup _{0 \leqslant t \leqslant T}|| \mathbf{x}\left(t, \mathbf{x}_{1}\right)\right|_{\mathcal{W}}-\left|\mathbf{x}\left(t, \mathbf{x}_{2}\right)\right| \mathcal{W} \mid,
\end{aligned}
$$

where $T=\max \left\{T_{\mathbf{x}_{1}}, T_{\mathbf{x}_{2}}\right\}$ and lemma 2 is used on the last step of transformations, $\mathbf{x}_{1}, \mathbf{x}_{2} \in M$. Due to Lipschitz continuity of the system (3) solutions for any compact set of initial conditions $\mathcal{D} \subset M$ and any time $0 \leqslant T<+\infty$, there exist $K \in \mathbb{R}_{+}$, $L \in \mathbb{R}_{+}$such that

$$
\left|\mathbf{x}\left(t, \mathbf{x}_{1}\right)-\mathbf{x}\left(t, \mathbf{x}_{2}\right)\right| \leqslant K\left|\mathbf{x}_{1}-\mathbf{x}_{2}\right|,\left.|| \mathbf{x}\left(t, \mathbf{x}_{1}\right)\right|_{\mathcal{W}}-\left|\mathbf{x}\left(t, \mathbf{x}_{2}\right)\right|_{\mathcal{W}}|\leqslant L| \mathbf{x}_{1}-\mathbf{x}_{2} \mid,
$$


for all $0 \leqslant t \leqslant T$ and any $\mathbf{x}_{1}, \mathbf{x}_{2} \in \mathcal{D}$. For all $0<\rho \leqslant \rho_{\max }$ and for any compact $\mathcal{D} \subset M$ there exists $T_{\rho}=\sup _{\mathbf{x}_{0} \in \mathcal{D} \backslash B_{\rho}} T_{\mathbf{x}_{0}}$ with the property $T_{\rho}<+\infty$ (due to local repelling property of the set $\mathcal{U}$, for any $0<\rho \leqslant \rho_{\max }$ there exists $0<\rho^{\prime} \leqslant \rho$ such that trajectories initiated into the set $\mathcal{D} \backslash B_{\rho}$ never reach the set $B_{\rho^{\prime}}$, see also facts $1-3$ for details). Owning this we obtain

$$
\begin{aligned}
\left|v\left(\mathbf{x}_{1}\right)-v\left(\mathbf{x}_{2}\right)\right| & \leqslant \sup _{0 \leqslant t \leqslant T_{\rho}}|| \mathbf{x}\left(t, \mathbf{x}_{1}\right)|\mathcal{W}-| \mathbf{x}\left(t, \mathbf{x}_{2}\right)|\mathcal{W}| \\
& \leqslant L\left|\mathbf{x}_{1}-\mathbf{x}_{2}\right|
\end{aligned}
$$

for all $\mathbf{x}_{1}, \mathbf{x}_{2} \in \mathcal{D} \backslash B_{\rho}$, and the function $v$ is locally Lipschitz continuous on the set $\mathcal{D} \backslash B_{\rho}$ for any fixed $0<\rho \leqslant \rho_{\max }$. The function $v$ is not increasing on any trajectory of the system (3), indeed for any $\mathbf{x}_{0} \in M$ :

$$
v\left(\mathbf{x}\left(t, \mathbf{x}_{0}\right)\right)=\sup _{\tau \geqslant 0}\left|\mathbf{x}\left(\tau, \mathbf{x}\left(t, \mathbf{x}_{0}\right)\right)\right|_{\mathcal{W}}=\sup _{\tau \geqslant t}\left|\mathbf{x}\left(\tau, \mathbf{x}_{0}\right)\right|_{\mathcal{W}} \leqslant \sup _{\tau \geqslant 0}\left|\mathbf{x}\left(\tau, \mathbf{x}_{0}\right)\right|_{\mathcal{W}}=v\left(\mathbf{x}_{0}\right) .
$$

Now, define a new function for all $\mathbf{x}_{0} \in M$ :

$$
V\left(\mathbf{x}_{0}\right)=\sup _{t \geqslant 0}\left\{v\left(\mathbf{x}\left(t, \mathbf{x}_{0}\right)\right) k(t)\right\}
$$

where $k: \mathbb{R}_{+} \rightarrow \mathbb{R}_{+}$is a continuously differentiable function satisfying $0<\kappa_{1} \leqslant$ $k(t) \leqslant \kappa_{2}<+\infty$ and $\dot{k}(t) \geqslant \kappa_{3}(t)>0$ for all $t \geqslant 0$. An example of such function is

$$
k(t)=\left(\kappa_{1}+\kappa_{2} t\right)(1+t)^{-1}, \dot{k}(t)=\left(\kappa_{2}-\kappa_{1}\right)(1+t)^{-2} .
$$

The function $V$ has bounds $\kappa_{1}\left|\mathbf{x}_{0}\right|_{\mathcal{W}} \leqslant V\left(\mathbf{x}_{0}\right) \leqslant \kappa_{2} \phi\left(\left|\mathbf{x}_{0}\right|_{\mathcal{W}}+R\right)$ and $V(\mathbf{x})=0$ for all $\mathbf{x} \in \mathcal{W}$. Again, for any $\mathbf{x}_{0} \in M$ there exists $T_{\mathbf{x}_{0}} \in \mathbb{R}_{+}$such that $V\left(\mathbf{x}_{0}\right)=$ $\sup _{0 \leqslant t \leqslant T_{\mathbf{x}_{0}}}\left\{v\left(\mathbf{x}\left(t, \mathbf{x}_{0}\right)\right) k(t)\right\}$. This claim follows from the non strict decreasing of the function $v$. A possible choice is $T_{\mathbf{x}_{0}}=T_{\mathbf{x}_{0}, \kappa_{1} \kappa_{2}^{-1}\left|\mathbf{x}_{0}\right| \mathcal{W}}$ for $\mathbf{x}_{0} \in M \backslash \mathcal{W}$ and $T_{\mathbf{x}_{0}}=0$ for $\mathbf{x}_{0} \in \mathcal{W}$. Next, for all $\mathbf{x}_{1}, \mathbf{x}_{2} \in M$,

$$
\begin{aligned}
\left|V\left(\mathbf{x}_{1}\right)-V\left(\mathbf{x}_{2}\right)\right| & =\left|\sup _{t \geqslant 0}\left\{v\left(\mathbf{x}\left(t, \mathbf{x}_{1}\right)\right) k(t)\right\}-\sup _{t \geqslant 0}\left\{v\left(\mathbf{x}\left(t, \mathbf{x}_{2}\right)\right) k(t)\right\}\right| \\
& =\left|\sup _{0 \leqslant t \leqslant T}\left\{v\left(\mathbf{x}\left(t, \mathbf{x}_{1}\right)\right) k(t)\right\}-\sup _{0 \leqslant t \leqslant T}\left\{v\left(\mathbf{x}\left(t, \mathbf{x}_{2}\right)\right) k(t)\right\}\right| \\
& \leqslant \sup _{0 \leqslant t \leqslant T}\left|k(t)\left[v\left(\mathbf{x}\left(t, \mathbf{x}_{1}\right)\right)-v\left(\mathbf{x}\left(t, \mathbf{x}_{2}\right)\right)\right]\right| \\
& \leqslant \kappa_{2} \sup _{0 \leqslant t \leqslant T}\left|v\left(\mathbf{x}\left(t, \mathbf{x}_{1}\right)\right)-v\left(\mathbf{x}\left(t, \mathbf{x}_{2}\right)\right)\right|,
\end{aligned}
$$

where $T=\max \left\{T_{\mathbf{x}_{1}}, T_{\mathbf{x}_{2}}\right\}$. For all $0<\rho \leqslant \rho_{\max }$ and for any compact $\mathcal{D} \subset M$ there exists $T_{\rho}=\sup _{\mathbf{x}_{0} \in \mathcal{D} \backslash B_{\rho}} T_{\mathbf{x}_{0}}$ such that $T_{\rho}<+\infty$ and

$$
\begin{aligned}
\left|V\left(\mathbf{x}_{1}\right)-V\left(\mathbf{x}_{2}\right)\right| & \leqslant \kappa_{2} \sup _{0 \leqslant t \leqslant T_{\rho}}\left|v\left(\mathbf{x}\left(t, \mathbf{x}_{1}\right)\right)-v\left(\mathbf{x}\left(t, \mathbf{x}_{2}\right)\right)\right| \\
& \leqslant \kappa_{2} L\left|\mathbf{x}\left(t, \mathbf{x}_{1}\right)-\mathbf{x}\left(t, \mathbf{x}_{2}\right)\right| \leqslant \kappa_{2} L K\left|\mathbf{x}_{1}-\mathbf{x}_{2}\right|
\end{aligned}
$$

for all $\mathbf{x}_{1}, \mathbf{x}_{2} \in \mathcal{D} \backslash B_{\rho}$. The function $V$ is locally Lipschitz continuous on the set $M \backslash B_{\rho}$ for any $0<\rho \leqslant \rho_{\max }$ and strictly decreasing for any $\mathbf{x}_{0} \in M \backslash \mathcal{W}$ :

$$
\begin{aligned}
V\left(\mathbf{x}\left(t, \mathbf{x}_{0}\right)\right) & =\sup _{\tau \geqslant 0}\left\{v\left(\mathbf{x}\left[\tau, \mathbf{x}\left(t, \mathbf{x}_{0}\right)\right]\right) k(\tau)\right\}=\sup _{\tau \geqslant t}\left\{v\left(\mathbf{x}\left[\tau, \mathbf{x}_{0}\right]\right) k(\tau-t)\right\} \\
& <\sup _{\tau \geqslant 0}\left\{v\left(\mathbf{x}\left[\tau, \mathbf{x}_{0}\right]\right) k(\tau)\right\}=V\left(\mathbf{x}_{0}\right),
\end{aligned}
$$


$V(t)$ equals zero on all trajectories in the set $\mathcal{W}$. Denote $L_{\mathbf{f}\left(\mathbf{x}_{0}\right)} V\left(\mathbf{x}_{0}\right)=\lim _{h \rightarrow 0} h^{-1}\left[V\left(\mathbf{x}\left(h, \mathbf{x}_{0}\right)\right)-\right.$ $\left.V\left(\mathbf{x}_{0}\right)\right]$, then

$$
L_{\mathbf{f}\left(\mathbf{x}_{0}\right)} V\left(\mathbf{x}_{0}\right)<0
$$

for a.e. $\mathbf{x}_{0} \in M \backslash \mathcal{W}$. Take some $r>\rho_{\max }$ such that the set $D_{r}=\{\mathbf{x} \in M$ : $\left.|\mathbf{x}|_{\mathcal{W}}<r\right\}$ is connected and globally attracting, then $T_{\rho}=\sup _{\mathbf{x}_{0} \in D_{r} \backslash B_{\rho}} T_{\mathbf{x}_{0}}$ and by definition

$$
\begin{aligned}
V\left(\mathbf{x}\left(h, \mathbf{x}_{0}\right)\right) & =\sup _{t \geqslant 0}\left\{v\left(\mathbf{x}\left[t, \mathbf{x}\left(h, \mathbf{x}_{0}\right)\right]\right) k(t)\right\}=\sup _{h \leqslant t \leqslant T_{\rho}}\left\{v\left(\mathbf{x}\left[t, \mathbf{x}_{0}\right]\right) k(t-h)\right\} \\
& =\sup _{0 \leqslant t \leqslant T_{\rho}}\left\{v\left(\mathbf{x}\left[t, \mathbf{x}_{0}\right]\right) K(t, h)\right\}, K(t, h)=\operatorname{if}[t<h, 0, k(t-h)],
\end{aligned}
$$

for a.e. $\mathbf{x}_{0} \in D_{r} \backslash\left(B_{\rho} \cup \mathcal{W}\right)$. Further

$$
\begin{aligned}
\sup _{0 \leqslant t \leqslant T_{\rho}}\left\{v\left(\mathbf{x}\left[t, \mathbf{x}_{0}\right]\right) K(t, h)\right\} & \leqslant V\left(\mathbf{x}_{0}\right) \sup _{0 \leqslant t \leqslant T_{\rho}}\left\{k(t)^{-1} K(t, h)\right\} \\
& =V\left(\mathbf{x}_{0}\right) \max \left\{0, \sup _{h \leqslant t \leqslant T_{\rho}}\left\{k(t)^{-1} k(t-h)\right\}\right\} \\
& =V\left(\mathbf{x}_{0}\right) \sup _{h \leqslant t \leqslant T_{\rho}}\left\{k(t)^{-1} k(t-h)\right\}
\end{aligned}
$$

and

$$
\begin{aligned}
\lim _{h \rightarrow 0} h^{-1}\left[V\left(\mathbf{x}\left(h, \mathbf{x}_{0}\right)\right)-V\left(\mathbf{x}_{0}\right)\right] & =\lim _{h \rightarrow 0} h^{-1}\left[V\left(\mathbf{x}_{0}\right) \sup _{h \leqslant t \leqslant T_{\rho}}\left\{k(t)^{-1} k(t-h)\right\}-V\left(\mathbf{x}_{0}\right)\right] \\
& =V\left(\mathbf{x}_{0}\right) \lim _{h \rightarrow 0} h^{-1}\left[\sup _{h \leqslant t \leqslant T_{\rho}}\left\{k(t)^{-1} k(t-h)\right\}-1\right] \\
& =V\left(\mathbf{x}_{0}\right) \lim _{h \rightarrow 0} h^{-1} \sup _{h \leqslant t \leqslant T_{\rho}} k(t)^{-1}\{k(t-h)-k(t)\} \\
& \leqslant V\left(\mathbf{x}_{0}\right) \sup _{0 \leqslant t \leqslant T_{\rho}} k(t)^{-1} \lim _{h \rightarrow 0} h^{-1}\{k(t-h)-k(t)\} \\
& =V\left(\mathbf{x}_{0}\right) \sup _{t \geqslant 0}\left\{-k(t)^{-1} \dot{k}(t)\right\} \leqslant-\kappa_{2}^{-1} \kappa_{3}\left(T_{\rho}\right) V\left(\mathbf{x}_{0}\right) .
\end{aligned}
$$

We approve the inequality

$$
L_{\mathbf{f}(\mathbf{x})} V(\mathbf{x}) \leqslant-\kappa_{2}^{-1} \kappa_{3}\left(T_{\rho}\right) V(\mathbf{x})
$$

for a.e. $\mathbf{x} \in D_{r} \backslash B_{\rho}$ (the inequality is additionally valid on the set $\mathcal{A}$ ). Now, consider the set $M \backslash D_{r}$. The time $T_{\mathbf{x}_{0}}=T_{\mathbf{x}_{0}, r}=\gamma\left(\left|\mathbf{x}_{0}\right| \mathcal{W}\right)$ is required for a trajectory $\mathbf{x}\left(t, \mathbf{x}_{0}\right), \mathbf{x}_{0} \in M \backslash D_{r}$ to reach the set $D_{r}$, where $\gamma$ is an increasing continuous function of initial distance $\left|\mathbf{x}_{0}\right| \mathcal{W}$ (see fact 2 for details). Thus $T_{\mathbf{x}_{0}} \leqslant \gamma\left[\kappa_{1}^{-1} V\left(\mathbf{x}_{0}\right)\right]$ and applying the same arguments as in the previous case for a.e. $\mathbf{x}_{0} \in M \backslash D_{r}$ we obtain:

$$
L_{\mathbf{f}(\mathbf{x})} V(\mathbf{x}) \leqslant-\kappa_{2}^{-1} \kappa_{3}\left(\gamma\left[\kappa_{1}^{-1} V(\mathbf{x})\right]\right) V(\mathbf{x}) .
$$

Combination of the inequalities (6) and (7) results in

$$
L_{\mathbf{f}(\mathbf{x})} V(\mathbf{x}) \leqslant-\alpha_{3}(V(\mathbf{x})), \alpha_{3}(s)=\kappa_{2}^{-1} \min \left\{\kappa_{3}\left(T_{\rho}\right), \kappa_{3}\left(\gamma\left[\kappa_{1}^{-1} s\right]\right),\right\} s
$$

for a.e. $\mathbf{x} \in M \backslash B_{\rho}$ and $\alpha_{1}(s)=\kappa_{1} s, \alpha_{2}(s)=\kappa_{2} \phi(s)$.

Lemma 4. Let all conditions of theorem 2 hold. Then for all $0<\rho<\rho_{\max }$ there exists a locally Lipschitz continuous function $V: B_{\rho} \rightarrow \mathbb{R}_{+}$(continuous on the set $\mathcal{U})$ such that for a.e. $|\mathbf{x}|_{\mathcal{U}} \leqslant \rho$ :

$$
\alpha_{1}\left(|\mathbf{x}|_{\mathcal{U}}\right) \leqslant V(\mathbf{x}) \leqslant \alpha_{2}\left(|\mathbf{x}|_{\mathcal{U}}\right), L_{\mathbf{f}(\mathbf{x})} V(\mathbf{x}) \geqslant \alpha_{3}(V(\mathbf{x})), \alpha_{3} \in \mathcal{K} .
$$


Proof. For $0<\rho<\rho_{\max }$ and all $\mathbf{x}_{0} \in B_{\rho} \backslash \mathcal{U}$ there exists $T_{\mathbf{x}_{0}} \in \mathbb{R}_{+}$such that $\mathbf{x}\left(t, \mathbf{x}_{0}\right) \notin B_{\rho}$ for all $t \geqslant T_{\mathbf{x}_{0}}$. Define

$$
v\left(\mathbf{x}_{0}\right)=\inf _{0 \leqslant t \leqslant T_{\mathbf{x}_{0}}}\left|\mathbf{x}\left(t, \mathbf{x}_{0}\right)\right|_{\mathcal{U}},
$$

by construction $\delta\left(\left|\mathbf{x}_{0}\right| \mathcal{U}\right) \leqslant v\left(\mathbf{x}_{0}\right) \leqslant\left|\mathbf{x}_{0}\right|_{\mathcal{U}}, \delta(s)=\left.s(1+s)^{-1} \inf _{\mid \mathbf{x}}\right|_{\mathcal{U}=s} v(\mathbf{x}), \delta \in \mathcal{K}$ and $v(\mathbf{x})=0$ for $\mathbf{x} \in \mathcal{U}$. To analyze continuity property of the function $v$, consider

$$
\begin{gathered}
\left|v\left(\mathbf{x}_{1}\right)-v\left(\mathbf{x}_{2}\right)\right|=\left|\inf _{0 \leqslant t \leqslant T_{\mathbf{x}_{1}}}\right| \mathbf{x}\left(t, \mathbf{x}_{1}\right)\left|\mathcal{U}-\inf _{0 \leqslant t \leqslant T_{\mathbf{x} 2}}\right| \mathbf{x}\left(t, \mathbf{x}_{2}\right)|\mathcal{U}| \\
\leqslant \sup _{0 \leqslant t \leqslant T}|| \mathbf{x}\left(t, \mathbf{x}_{1}\right)|\mathcal{U}-| \mathbf{x}\left(t, \mathbf{x}_{2}\right)|\mathcal{U}|,
\end{gathered}
$$

where $T=\max \left\{T_{\mathbf{x}_{1}}, T_{\mathbf{x}_{2}}\right\}$ and the last step follows lemma 2. Due to Lipschitz continuity of the system (3) solutions for any compact set of initial conditions $\mathcal{D} \subset M$ and time $0 \leqslant T<+\infty$, there exist $K \in \mathbb{R}_{+}, L \in \mathbb{R}_{+}$such that

$$
\left|\mathbf{x}\left(t, \mathbf{x}_{1}\right)-\mathbf{x}\left(t, \mathbf{x}_{2}\right)\right| \leqslant K\left|\mathbf{x}_{1}-\mathbf{x}_{2}\right|,|| \mathbf{x}\left(t, \mathbf{x}_{1}\right)|\mathcal{U}-| \mathbf{x}\left(t, \mathbf{x}_{2}\right)|\mathcal{U}| \leqslant L\left|\mathbf{x}_{1}-\mathbf{x}_{2}\right|,
$$

for all $0 \leqslant t \leqslant T$ and any $\mathbf{x}_{1}, \mathbf{x}_{2} \in \mathcal{D}$. For all $0<\rho^{\prime}<\rho$ and $D_{r}=\left\{\mathbf{x} \in M:|\mathbf{x}|_{\mathcal{W}}<\right.$ $r\}$ with $r \geqslant \rho$, there exists $T_{\rho^{\prime}, r}=\sup _{\mathbf{x}_{0} \in B_{\rho} \backslash B_{\rho^{\prime}} \cap D_{r}} T_{\mathbf{x}_{0}}$ satisfying $T_{\rho^{\prime}, r}<+\infty$ and $T_{\rho^{\prime}, r} \rightarrow+\infty$ with $\rho^{\prime} \rightarrow 0$. This implies

$$
\left|v\left(\mathbf{x}_{1}\right)-v\left(\mathbf{x}_{2}\right)\right| \leqslant \sup _{0 \leqslant t \leqslant T_{\rho^{\prime}, r}}|| \mathbf{x}\left(t, \mathbf{x}_{1}\right)|\mathcal{U}-| \mathbf{x}\left(t, \mathbf{x}_{2}\right)|\mathcal{U}| \leqslant L\left|\mathbf{x}_{1}-\mathbf{x}_{2}\right|
$$

for all $\mathbf{x}_{1}, \mathbf{x}_{2} \in B_{\rho} \backslash B_{\rho^{\prime}} \cap D_{r}$, and the function $v$ is locally Lipschitz continuous on the set $B_{\rho} \backslash B_{\rho^{\prime}}$ for any fixed $0<\rho^{\prime}<\rho$. Therefore, $v$ is locally Lipschitz continuous on $B_{\rho} \backslash \mathcal{U}$ and continuous on $B_{\rho}$. The function $v$ is not decreasing on any trajectory of the system (3), indeed for any $\mathbf{x}_{0} \in B_{\rho}$ :

$$
\begin{aligned}
v\left(\mathbf{x}\left(t, \mathbf{x}_{0}\right)\right) & =\inf _{0 \leqslant \tau \leqslant T_{\mathbf{x}\left(t, \mathbf{x}_{0}\right)}}\left|\mathbf{x}\left(\tau, \mathbf{x}\left(t, \mathbf{x}_{0}\right)\right)\right|_{\mathcal{U}} \\
& =\inf _{t \leqslant \tau \leqslant T_{\mathbf{x}_{0}}}\left|\mathbf{x}\left(\tau, \mathbf{x}_{0}\right)\right|_{\mathcal{U}} \\
& \geqslant \inf _{0 \leqslant \tau \leqslant T_{\mathbf{x}_{0}}}\left|\mathbf{x}\left(\tau, \mathbf{x}_{0}\right)\right|_{\mathcal{U}}=v\left(\mathbf{x}_{0}\right) .
\end{aligned}
$$

Now, define a new function for all $\mathbf{x}_{0} \in B_{\rho}$ :

$$
V\left(\mathbf{x}_{0}\right)=\inf _{0 \leqslant t \leqslant T_{\mathbf{x}_{0}}}\left\{v\left(\mathbf{x}\left(t, \mathbf{x}_{0}\right)\right) k(t)\right\},
$$

where $k: \mathbb{R}_{+} \rightarrow \mathbb{R}_{+}$is a continuously differentiable function with properties $0<$ $\kappa_{1} \leqslant k(t) \leqslant \kappa_{2}<+\infty$ and $\dot{k}(t) \leqslant-\kappa_{3}(t)<0$ for all $t \geqslant 0$. An example of such function is

$$
k(t)=\kappa_{1}+\left(\kappa_{2}-\kappa_{1}\right) e^{-t}, \dot{k}(t)=\left(\kappa_{1}-\kappa_{2}\right) e^{-t} .
$$

The function $V$ has bounds $\kappa_{1} \delta\left(\left|\mathbf{x}_{0}\right| \mathcal{W}\right) \leqslant V\left(\mathbf{x}_{0}\right) \leqslant \kappa_{2}\left|\mathbf{x}_{0}\right| \mathcal{W}$ and $V(\mathbf{x})=0$ for all $\mathbf{x} \in \mathcal{U}$. Next, for all $\mathbf{x}_{1}, \mathbf{x}_{2} \in B_{\rho}$,

$$
\begin{aligned}
\left|V\left(\mathbf{x}_{1}\right)-V\left(\mathbf{x}_{2}\right)\right| & =\left|\inf _{0 \leqslant t \leqslant T_{\mathbf{x}_{1}}}\left\{v\left(\mathbf{x}\left(t, \mathbf{x}_{1}\right)\right) k(t)\right\}-\inf _{0 \leqslant t \leqslant T_{\mathbf{x}_{0}}}\left\{v\left(\mathbf{x}\left(t, \mathbf{x}_{2}\right)\right) k(t)\right\}\right| \\
& =\left|\inf _{0 \leqslant t \leqslant T}\left\{v\left(\mathbf{x}\left(t, \mathbf{x}_{1}\right)\right) k(t)\right\}-\inf _{0 \leqslant t \leqslant T}\left\{v\left(\mathbf{x}\left(t, \mathbf{x}_{2}\right)\right) k(t)\right\}\right| \\
& \leqslant \sup _{0 \leqslant t \leqslant T}\left|k(t)\left[v\left(\mathbf{x}\left(t, \mathbf{x}_{1}\right)\right)-v\left(\mathbf{x}\left(t, \mathbf{x}_{2}\right)\right)\right]\right| \\
& \leqslant \kappa_{2} \sup _{0 \leqslant t \leqslant T}\left|v\left(\mathbf{x}\left(t, \mathbf{x}_{1}\right)\right)-v\left(\mathbf{x}\left(t, \mathbf{x}_{2}\right)\right)\right|,
\end{aligned}
$$


where $T=\max \left\{T_{\mathbf{x}_{1}}, T_{\mathbf{x}_{2}}\right\}$ and lemma 2 is again used in the second step. For all $0<\rho^{\prime}<\rho$ and $D_{r}$ with $r \geqslant \rho$, there exists $T_{\rho^{\prime}, r}=\sup _{\mathbf{x}_{0} \in B_{\rho} \backslash B_{\rho^{\prime}} \cap D_{r}} T_{\mathbf{x}_{0}}<+\infty$ and

$$
\begin{gathered}
\left|V\left(\mathbf{x}_{1}\right)-V\left(\mathbf{x}_{2}\right)\right| \leqslant \kappa_{2} \sup _{0 \leqslant t \leqslant T_{\rho^{\prime}, r}}\left|v\left(\mathbf{x}\left(t, \mathbf{x}_{1}\right)\right)-v\left(\mathbf{x}\left(t, \mathbf{x}_{2}\right)\right)\right| \\
\leqslant \kappa_{2} L\left|\mathbf{x}\left(t, \mathbf{x}_{1}\right)-\mathbf{x}\left(t, \mathbf{x}_{2}\right)\right| \leqslant \kappa_{2} L K\left|\mathbf{x}_{1}-\mathbf{x}_{2}\right|
\end{gathered}
$$

for all $\mathbf{x}_{1}, \mathbf{x}_{2} \in B_{\rho} \backslash B_{\rho^{\prime}} \cap D_{r}$. Then the function $V$ is locally Lipschitz continuous on the set $B_{\rho} \backslash \mathcal{U}$ and continuous on $B_{\rho}$. This function is strictly increasing for any $\mathbf{x}_{0} \in B_{\rho} \backslash \mathcal{U}:$

$$
\begin{aligned}
V\left(\mathbf{x}\left(t, \mathbf{x}_{0}\right)\right) & =\inf _{0 \leqslant \tau \leqslant T_{\mathbf{x}\left(t, \mathbf{x}_{0}\right)}}\left\{v\left(\mathbf{x}\left[\tau, \mathbf{x}\left(t, \mathbf{x}_{0}\right)\right]\right) k(\tau)\right\} \geqslant \inf _{t \leqslant \tau \leqslant T_{\mathbf{x}_{0}}}\left\{v\left(\mathbf{x}\left[\tau, \mathbf{x}_{0}\right]\right) k(\tau-t)\right\} \\
& >\inf _{0 \leqslant \tau \leqslant T_{\mathbf{x}_{0}}}\left\{v\left(\mathbf{x}\left[\tau, \mathbf{x}_{0}\right]\right) k(\tau)\right\}=V\left(\mathbf{x}_{0}\right),
\end{aligned}
$$

$V(t)$ equals zero on any trajectories in the set $\mathcal{U}$, then

$$
L_{\mathbf{f}\left(\mathbf{x}_{0}\right)} V\left(\mathbf{x}_{0}\right)=\lim _{h \rightarrow 0} h^{-1}\left[V\left(\mathbf{x}\left(h, \mathbf{x}_{0}\right)\right)-V\left(\mathbf{x}_{0}\right)\right]>0
$$

for a.e. $\mathbf{x}_{0} \in B_{\rho} \backslash \mathcal{U}$. By definition

$$
\begin{aligned}
V\left(\mathbf{x}\left(h, \mathbf{x}_{0}\right)\right) & =\inf _{0 \leqslant t \leqslant T_{\mathbf{x}\left(h, \mathbf{x}_{0}\right)}}\left\{v\left(\mathbf{x}\left[t, \mathbf{x}\left(h, \mathbf{x}_{0}\right)\right]\right) k(t)\right\} \\
& =\inf _{h \leqslant t \leqslant T_{\mathbf{x}_{0}}}\left\{v\left(\mathbf{x}\left[t, \mathbf{x}_{0}\right]\right) k(t-h)\right\}=\inf _{h \leqslant t \leqslant T_{\mathbf{x}_{0}}}\left\{v\left(\mathbf{x}\left[t, \mathbf{x}_{0}\right]\right) k(t) k(t)^{-1} k(t-h)\right\} \\
& \geqslant \inf _{h \leqslant t \leqslant T_{\mathbf{x}_{0}}}\left\{v\left(\mathbf{x}\left[t, \mathbf{x}_{0}\right]\right) k(t)\right\} \inf _{h \leqslant t \leqslant T_{\mathbf{x}_{0}}}\left\{k(t)^{-1} k(t-h)\right\} \\
& \geqslant V\left(\mathbf{x}_{0}\right) \inf _{h \leqslant t \leqslant T_{\mathbf{x}_{0}}}\left\{k(t)^{-1} k(t-h)\right\} .
\end{aligned}
$$

Finally,

$$
\begin{aligned}
\lim _{h \rightarrow 0} h^{-1}\left[V\left(\mathbf{x}\left(h, \mathbf{x}_{0}\right)\right)-V\left(\mathbf{x}_{0}\right)\right] & \geqslant \lim _{h \rightarrow 0} h^{-1}\left[V\left(\mathbf{x}_{0}\right) \inf _{h \leqslant t \leqslant T_{\mathbf{x}_{0}}}\left\{k(t)^{-1} k(t-h)\right\}-V\left(\mathbf{x}_{0}\right)\right] \\
& =V\left(\mathbf{x}_{0}\right) \lim _{h \rightarrow 0} h^{-1}\left[\inf _{h \leqslant t \leqslant T_{\mathbf{x}_{0}}}\left\{k(t)^{-1} k(t-h)\right\}-1\right] \\
& =V\left(\mathbf{x}_{0}\right) \lim _{h \rightarrow 0} h^{-1} \inf _{h \leqslant t \leqslant T_{\mathbf{x}_{0}}} k(t)^{-1}\{k(t-h)-k(t)\} \\
& \geqslant V\left(\mathbf{x}_{0}\right) \inf _{0 \leqslant t \leqslant T_{\mathbf{x}_{0}}} k(t)^{-1} \lim _{h \rightarrow 0} h^{-1}\{k(t-h)-k(t)\} \\
& =V\left(\mathbf{x}_{0}\right) \inf _{0 \leqslant t \leqslant T_{\mathbf{x}_{0}}}\left\{-k(t)^{-1} \dot{k}(t)\right\} \geqslant \kappa_{2}^{-1} \kappa_{3}\left(T_{\mathbf{x}_{0}}\right) V\left(\mathbf{x}_{0}\right) .
\end{aligned}
$$

We substantiate the inequality

$$
L_{\mathbf{f}(\mathbf{x})} V(\mathbf{x}) \geqslant \alpha_{3}(V(\mathbf{x})), \alpha_{3}(s)=\kappa_{2}^{-1} s \inf _{V(\mathbf{x}) \geqslant s \wedge \mathbf{x} \in B_{\rho}} \kappa_{3}\left(T_{\mathbf{x}}\right)
$$

for a.e. $\mathbf{x} \in B_{\rho}$ (the inequality is additionally valid on the set $\left.\mathcal{U}\right), \alpha_{1}(s)=\kappa_{1} \delta(s)$, $\alpha_{2}(s)=\kappa_{2} s$.

Lemma 5. Let all conditions of theorem 2 hold. Then for all $0<\rho<\rho_{\max }$ and any $\gamma>0$, there exists a locally Lipschitz continuous function $V_{2}: B_{\rho} \rightarrow \mathbb{R}_{+}$ (continuous on the set $\mathcal{U}$ ) such that

$$
\gamma<V_{2}(\mathbf{x})<+\infty, L_{\mathbf{f}(\mathbf{x})} V_{2}(\mathbf{x}) \leqslant-\kappa(|\mathbf{x}| \mathcal{U}) \alpha\left(V_{2}(\mathbf{x})\right), \kappa, \alpha \in \mathcal{K}
$$

for a.e. $|\mathbf{x}|_{\mathcal{U}} \leqslant \rho$. 

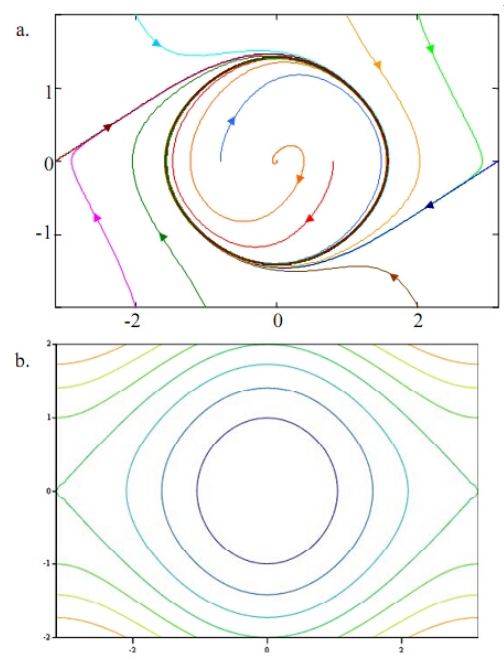

FiguRE 5. Trajectories and Lyapunov function contours for the system (8)

Proof. Since all conditions of lemma 4 are satisfied, for all $0<\rho<\rho_{\max }$, there exists a locally Lipschitz continuous function $V: B_{\rho} \rightarrow \mathbb{R}_{+}$(continuous on the set $\mathcal{U})$ such that $\alpha_{1}\left(|\mathbf{x}|_{\mathcal{U}}\right) \leqslant V(\mathbf{x}) \leqslant \alpha_{2}\left(|\mathbf{x}|_{\mathcal{U}}\right), L_{\mathbf{f}(\mathbf{x})} V(\mathbf{x}) \geqslant \alpha_{3}(V(\mathbf{x})), \alpha_{3} \in \mathcal{K}$ for a.e. $|\mathbf{x}|_{\mathcal{U}} \leqslant \rho$. Define $V_{2}(\mathbf{x})=v-V(\mathbf{x})$ where $\gamma+\alpha_{2}\left(\rho_{\max }\right)<v<+\infty$, then $\gamma<V_{2}(\mathbf{x})<+\infty$ and

$$
L_{\mathbf{f}(\mathbf{x})} V_{2}(\mathbf{x})=-L_{\mathbf{f}(\mathbf{x})} V(\mathbf{x}) \leqslant-\alpha_{3}(V(\mathbf{x})) .
$$

Since $V_{2}(\mathbf{x}) \leqslant v$ for all $\mathbf{x} \in B_{\rho}$, then

$$
L_{\mathbf{f}(\mathbf{x})} V_{2}(\mathbf{x}) \leqslant-\alpha_{3}(V(\mathbf{x})) \leqslant-\alpha_{3} \circ \alpha_{1}(|\mathbf{x}| \mathcal{U}) \leqslant-v^{-1} \alpha_{3} \circ \alpha_{1}(|\mathbf{x}| \mathcal{U}) V_{2}(\mathbf{x})
$$

and the result follows for $\kappa(s)=\alpha_{3} \circ \alpha_{1}(s), \alpha(s)=s / v$.

\section{EXAMPLes}

The third example is a controlled conservative pendulum [25]:

$$
\begin{gathered}
\dot{x}_{1}=x_{2} ; \\
\dot{x}_{2}=-\omega^{2} \sin \left(x_{1}\right)+u\left(x_{1}, x_{2}\right) ; \\
u\left(x_{1}, x_{2}\right)=-x_{2}\left[H\left(x_{1}, x_{2}\right)-H^{*}\right], H\left(x_{1}, x_{2}\right)=0.5 x_{2}^{2}+\omega^{2}\left[1-\cos \left(x_{1}\right)\right],
\end{gathered}
$$

where $x_{1} \in[-\pi, \pi)$ is the angle coordinate, $x_{2} \in \mathbb{R}$ is the angle velocity, $\omega \in \mathbb{R}$ is the natural frequency of the pendulum, $u \in \mathbb{R}$ is the controlling input, $H$ is the Hamiltonian function of the unforced pendulum (8), $\dot{H}=-x_{2}^{2}\left[H\left(x_{1}, x_{2}\right)-H^{*}\right]$, $H^{*} \in\left(0, \omega^{2}\right)$ is the stabilized level of Hamiltonian function. The system (8) has two equilibriums $(0,0),(\pi, 0)$ and asymptotically stable limit cycle corresponding to $\Gamma=\left\{\left(x_{1}, x_{2}\right): H\left(x_{1}, x_{2}\right)=H^{*}\right\}$. Linearization of the system (8) shows that both equilibriums are unstable (the first one has two complex conjugate eigenvalues with positive real parts, the second has two real eigenvalues, one is negative and another is positive). The set of all invariant solutions of this system $\mathcal{W}$ is composed by these equilibriums and the limit cycle, i.e., $\mathcal{W}=\{(0,0),(\pi, 0) \cup \Gamma\}$. This set is 

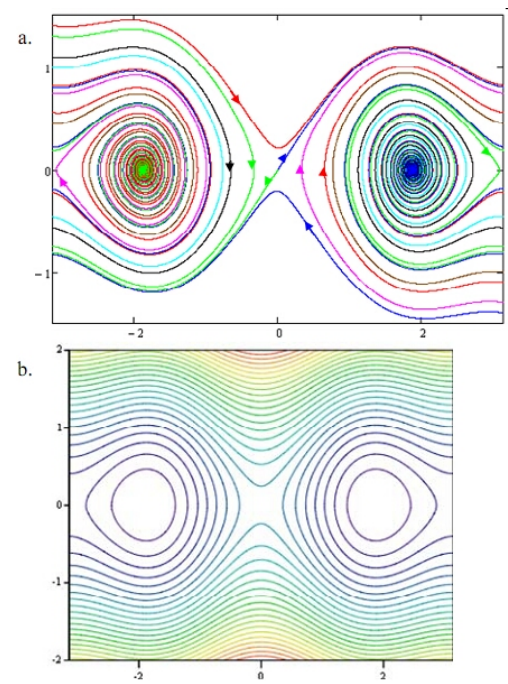

FiguRE 6. Trajectories and Lyapunov function contours for the system (9)

globally attractive (trajectories of the system (8) for $\omega=1$ are plotted in Fig. 5,a). The system (8) has Lyapunov function

$$
W\left(x_{1}, x_{2}\right)=0.5\left[H\left(x_{1}, x_{2}\right)-H^{*}\right]
$$

which equals zero on the limit cycle $\Gamma$ and is positive otherwise (the function $W$ contour plot is shown in Fig. 5,b). The time derivative of $W$ for the system (8) is not positive:

$$
\dot{W}=-y^{2}, y=x_{2}\left[H\left(x_{1}, x_{2}\right)-H^{*}\right] .
$$

All conditions of theorem 2 hold and this system has $\mathcal{W}$-Lyapunov function from definition 1 and the corresponding $\mathcal{K} \mathcal{L}$ estimate from proposition 2.

Finally, consider a mass $m$ along a circle of radius $a$. The circle rotates about its vertical diameter with constant angular velocity $\Omega$. The mass is subject to gravity and friction, the equation of motion is

$$
m a^{2} \ddot{\theta}=m \Omega^{2} a^{2} \sin (\theta) \cos (\theta)-m g a \sin (\theta)-k \dot{\theta},
$$

where $\theta \in[-\pi, \pi)$ is the angular position of the mass and $k>0$ is the coefficient of friction. Introducing the dimensionless parameters $\lambda=g /\left(\Omega^{2} a\right)$ and $\kappa=k /\left(m a \Omega^{2}\right)$, new coordinates $x_{1}=\theta, x_{2}=\dot{\theta}$ and performing a change of time $t^{\prime}=\Omega t$, we obtain the following equation of motion:

$$
\dot{x}_{1}=x_{2}, \dot{x}_{2}=\left[\cos \left(x_{1}\right)-\lambda\right] \sin \left(x_{1}\right)-\kappa x_{2} .
$$

For $-1<\lambda<0$, this system has four equilibriums, and the set

$$
\mathcal{W}=\{(0,0),(-\pi, 0),(\arccos (\lambda), 0),(-\arccos (\lambda), 0)\} .
$$

Linearization shows that the first two equilibriums are saddle points and the last two are stable focuses. The system has a Lyapunov function

$$
W\left(x_{1}, x_{2}\right)=0.5 x_{2}^{2}+V\left(x_{1}\right)-V(\arccos (\lambda)),
$$


where $V\left(x_{1}\right)=-\lambda \cos \left(x_{1}\right)-.5 \sin \left(x_{1}\right)^{2}$ is the system (9) potential. The Lyapunov function equals zero in the stable equilibriums only and it is positive otherwise, its time derivative has form

$$
\dot{W}=-\kappa y^{2}, y=x_{2} .
$$

The system is $\mathcal{W}$-detectable with respect to the output $y$ (the system trajectories and the contour levels of the Lyapunov function for $\lambda=-0.3, \kappa=0.1$ are plotted in Fig. 6). Again, all conditions of theorem 2 hold and the system (9) has $\mathcal{W}$-Lyapunov function from definition 1 and the corresponding $\mathcal{K} \mathcal{L}$ estimate from proposition 2.

\section{Conclusion}

The paper presents a new global stability definition covering the case of multistable systems. Necessary and sufficient characterizations in terms of Lyapunov functions are proposed. Useful $\mathcal{K} \mathcal{L}$ estimates on systems solutions are computed. We show that several well known in the literature examples of multi-stable systems satisfy this new stability property. Availability of the corresponding Lyapunov functions for these examples is established.

The proposed stability definition is rather generic. For instance, consider a chaotic system having invariant solutions presented by a set of equilibriums (stable/unstable/hyperbolic) and by a compact strange attractor. It could be used as a target for the proposed concept in order to analyze the system behavior globally from its multi-stability perspective. In this case, even chaotic systems have to possess the $\mathcal{K} \mathcal{L}$ estimates from proposition 2 and the corresponding Lyapunov functions from theorem 2.

Global nature of stability property and some properties of Lyapunov functions may help in robustness analysis with respect to external disturbances, which is a future direction of research. Also, the conservatism of conditions can be relaxed in future works.

\section{ACKNOWLEDGMENTS}

The author would like to thank R. Sepulchre and D. Berdjag for helpful discussions and comments.

\section{REFERENCES}

[1] D. Angeli. An almost global notion of input-to-state stability. IEEE Trans. Automatic Control, 49:866-874, 2004.

[2] D. Angeli, J.E. Ferrell, and E.D. Sontag. Detection of multistability, bifurcations and hysteresis in a large class of biological positive-feedback systems. Proc. Natl. Acad. Sci. USA, 101:1822-1827, 2004.

[3] D. Angeli and E.D. Sontag. Multi-stability in monotone input/output systems. SystemsEC Control Lett., 51:185-202, 2004.

[4] D. Angeli and E.D. Sontag. Oscillations in i/o monotone systems. IEEE Trans. Circ. Systems, $55: 166-176,2008$.

[5] D.V. Anosov. Geodesic flows on Riemannian manifolds with negative curvature, volume 90 of American Mathematical Society. Providence, 1967.

[6] D.V. Anosov, editor. Dynamical systems, volume 66 of Encyclopedia of mathematical science. Birkhäuser, 1995.

[7] M. Chaves, T. Eissing, and F. Allgower. Bistable biological systems: A characterization through local compact input-to-state stability. IEEE Trans. Automatic Control, 45:87-100, 2008.

[8] D.V. Efimov and A.L. Fradkov. Oscillatority of nonlinear systems with static feedback. SIAM Journal on Optimization and Control, 48(2):618-640, 2009. 
[9] G. Enciso and E.D. Sontag. Monotone systems under positive feedback: multistability and a reduction theorem. Systems\& Control Lett., 54:159-168, 2005.

[10] A.Kh. Gelig, G.A. Leonov, and V.A. Yakubovich. Stability of nonlinear systems with non unique equilibrium. Nauka, Moscow, 1978. [in Russian].

[11] W. Hahn. Stability of Motion. SpringerWerlag, 1967.

[12] C. Hayachi. Nonlinear oscillations in physical systems. McGraw-Hill Book Company, New York, 1964.

[13] H.K. Khalil. Nonlinear Systems. Macmillan, 1992.

[14] V. Lakshmikantham and X.Z. Liu. Stability analysis in terms of two measures. World Scientific, 1993.

[15] M. Laurent and N. Kellershohn. Multistability: a major means of differentiation and evolution in biological systems. Trends Biochem. Sci., 24:418-422, 1999.

[16] Y. Lin, E.D. Sontag, and Y. Wang. A smooth converse Lyapunov theorem for robust stability. SIAM J. Control and Optimization, 34:124-160, 1996.

[17] A.M. Lyapunov. The general problem of the stability of motion. Taylor \& Francis, London, 1992.

[18] P. Monzón and R. Potrie. Local and global aspects of almost global stability. In Proc. 45th IEEE Conf. on Decision and Control, pages 5120-5125, San Diego, USA, 2006.

[19] Z. Nitecki and M. Shub. Filtrations, decompositions, and explositions. American Journal of Mathematics, 97(4):1029-1047, 1975.

[20] C. Pugh and M. Shub. Omega stability theorem for flows. Inventiones Math, 11:150-158, 1970.

[21] R. Rajaram, U. Vaidya, and M. Fardad. Connection between almost everywhere stability of an ode and advection pde. In Proc. 46th IEEE Conf. Decision and Control, pages 5880-5885, New Orleans, 2007.

[22] A. Rantzer. A dual to Lyapunov's stability theorem. Syst. Control Lett., 42:161-168, 2001.

[23] V.V. Rumyantsev and A.S. Oziraner. Stability and stabilization of motion with respect to part of variables. Nauka, Moscow, 1987. [in Russian].

[24] R. Sepulchre, M. Jankovic, and P. Kokotoviã. Constructive nonlinear control. Springer-Verlag, New York, 1997.

[25] A.S. Shiriaev and A.L. Fradkov. Stabilization of invariant sets for nonlinear non-affine systems. Automatica, 36:1709-1715, 2000.

[26] S. Smale. Differentiable dynamical systems. Bull. Amer. Math. Soc., 73:747-817, 1967.

[27] E.D. Sontag. On the input-to-state stability property. European J. Control, 1:24-36, 1995.

[28] E.D. Sontag. Comments on integral variants of iss. Systems\& Control Lett., 34:93-100, 1998.

[29] E.D. Sontag and Y. Wang. Notions of input to output stability. Systems\&Control Lett., 38:235-248, 1999.

[30] E.D. Sontag and Y. Wang. Lyapunov characterizations of input to output stability. SIAM J. Control and Optimization, 39:226-249, 2001.

[31] G.-B. Stan and R. Sepulchre. Analysis of interconnected oscillators by dissipativity theory. IEEE Trans. Automatic Control, 52:256-270, 2007.

[32] A. Teel and L. Praly. A smooth Lyapunov function from a class-kl estimate involving two positive semidefinite functions. ESAIM: Control, Optimisation and Calculus of Variations, $5: 313-367,2000$.

[33] R. van Handel. Almost global stochastic stability. SIAM J. Control and Optimization, 45(4):1297-1313, 2006.

[34] V.I. Vorotnikov. Partial Stability and Control. Birkhauser, Boston, 1998.

[35] F. Wesley Jr. Wilson. Smoothing derivatives of functions and applications. Technical report, Brown Univ. Providence, Div. of Applied Mathematics, 1966.

Non-A project at inRia - Lne, Parc Scientifique de la Haute Borne, 40 avenue Halley, Bât. A Park Plaza 59650 Villeneuve d'Ascq, France. On leaving Systems and Control @ Systems and Modeling, Department of Electrical Engineering and Computer Science, B28 Université de Liège, B-4000 Liege Sart-Tilman, Belgium.

E-mail address: Denis.Efimov@inria.fr 